Research Article

\title{
New Finite Modeling of Free and Forced Vibration Responses of Piezoelectric FG Plates Resting on Elastic Foundations in Thermal Environments
}

\author{
Pham Minh Phuc $\mathbb{D}$ and Nguyen Thi Kim Khue \\ Faculty of Basic Sciences, University of Transport and Communications, 03 Cau Giay Street, Dong Da, Hanoi, Vietnam \\ Correspondence should be addressed to Pham Minh Phuc; bmclt@utc.edu.vn
}

Received 4 December 2020; Revised 4 January 2021; Accepted 19 January 2021; Published 2 February 2021

Academic Editor: Nerio Tullini

Copyright (c) 2021 Pham Minh Phuc and Nguyen Thi Kim Khue. This is an open access article distributed under the Creative Commons Attribution License, which permits unrestricted use, distribution, and reproduction in any medium, provided the original work is properly cited.

\begin{abstract}
This paper carries out free and forced vibration analysis of piezoelectric FGM plates resting on two-parameter elastic foundations placed in thermal environments. By employing the third-order shear deformation theory and the finite element method, this work establishes free and forced vibration equations of piezoelectric FGM plates, where the materials are assumed to be varied in the thickness directions, and the mechanical properties depend on the temperature. Then, comparative examples are conducted to verify the proposed theory and mathematical model, and the results of this study and other methods meet a very good agreement. Then, effects of geometrical and material properties such as the feedback coefficient, voltage, volume fraction index, temperature as well as the parameters of elastic foundations on free and forced vibration of the plates are investigated, and the conclusions are given out to provide the effective direction for the design and practical use of these structures.
\end{abstract}

\section{Introduction}

Nowadays, due to the development of science and technology, complicated smart mechanical structures are used more and more in engineering practice, where they are integrated with numerous kinds of materials such as composite materials, functionally graded materials (FGM), piezoelectric layers, magnetorheological materials, and shape memory alloy. Therefore, mechanical investigations of these structures need to use advanced approaches and cost much time to deal with. Besides, mechanical systems in many cases can be rested on elastic structures, so it can seem to be elastic foundations; thus, scientists tend to examine mechanical responses of these structures lying on other elastic parts, and these problems are more and more challenging. In this section, a review of related works is firstly carried out to find out the lack of published papers as well as figure the reason to conduct this investigation.

For investigations relating to piezoelectric FGM plates, some spotlight papers can be counted as follows. Lieu et al.
[1] for the first time introduced an isogeometric Bézier FE formulation to conduct the static bending and transient analysis of piezoelectric functionally graded porous (FGP) plates reinforced by graphene platelets (GPLs), in which the modified Halpin-Tsai micromechanical model was employed to evaluate the effective mechanical characteristics which varied gradually in the thickness direction of the core layer, and the electric potential was assumed to change linearly through the thickness for each piezoelectric sublayer. Barati and colleagues [2] investigated free vibration of piezoelectric FGM plates with porosities by using a refined four-unknown plate theory, which could capture shear deformation impacts needless of the shear correction factor. A modified power-law model was assumed to describe the changing rule of graded material properties of a piezoelectric functionally graded plate. Governing equations derived from Hamilton's principle was solved by an analytical method, which satisfied several kinds of boundary conditions. Nam et al. [3] studied free and forced vibration of smart FG metal foam plate structures reinforced by graphene platelets 
(GPLs). They then examined the active control of FG metal foam plates with piezoelectric layers. A computational approach based on a generalized-type higher-order shear deformation theory (CO-HSDT) polygonal finite element formulation (PFEM) was modified to provide numerical solutions. The numerical results showed that the proposed approach was suitable for modeling both thick and thin structures. Keleshteri and his coworkers [4] used the firstorder shear deformation theory (FSDT) and von Karman geometrical nonlinearity along with the Hamilton principle to study large-scale vibration response of piezoelectric FG carbon nanotube-reinforced composite (FG-CNTRC) annular sector plates. Malekzadeh et al. [5] developed a transformed differential quadrature method (TDQM) to investigate the free vibration response of FG multilayer nanocomposite eccentric annular plates. The modified Halpin-Tsai model was also used to estimate the effective mechanical properties of the nanocomposites. The governing equations were derived by using the FSDT and Hamilton's principle and Maxwell's equation. The numerical data pointed out that the TDQM met a very good convergence rate. Ebrahimi and Rastgoo [6] carried out a nonlinear free vibration analysis of thin annular piezoelectric functionally graded plates, where the material properties of the FG core plate were assumed to be changed in the thickness direction based on the power-law distribution. A good agreement between the results of their work and those of the finite element (FE) analyses were obtained. Keleshteri et al. [7, 8], respectively, presented buckling analysis and postbuckling analysis of piezoelectric FGCNTRC rectangular and annular plates.

For FGM plates resting on elastic foundations, scientists also have published many good works on these problems. Hassen et al. [9] examined the free vibration analysis of simply supported functionally graded plates (FGPs) resting on a Winkler-Pasternak elastic foundation using a new higher shear deformation theory. Chan et al. [10] carried out the nonlinear dynamic analysis of piezoelectric functionally graded porous truncated conical panel in thermal environments by using an analytical method, where the panel resting on an elastic foundation was modeled according to the Winkler-Pasternak theory. The material properties including Young's modulus, shear modulus, and density were assumed to gradually through the shell thickness. Theoretical formulations were presented based on the first-order shear deformation shell theory with a von Karman-Donnell type of kinematic nonlinearity. Zenkour and Radwan [11] studied the bending response of FG plates resting on elastic foundations in a hygrothermal environment with porosities. Shen and Xiang [12] introduced a postbuckling analysis of carbon nanotube-reinforced composite (CNTRC) cylindrical panels resting on elastic foundations and subjected to axial compression in thermal environments. The cylindrical panels were reinforced by aligned single-walled carbon nanotubes (SWCNTs) which were assumed to be functionally graded (FG) through the thickness direction with different types of distributions. The material properties of FG-CNTRC panels were evaluated through an extended rule of mixture micromechanical model. The governing equations were based on a higher-order shear deformation theory with a von Kármán type of kinematic nonlinearity. Ninh [13] examined nonlinear thermal torsional postbuckling of piezoelectric carbon nanotube-reinforced composite cylindrical shell surrounded by an elastic medium. Nebab et al. [14] used an analytical method to research wave propagation in simply supported graduated functional plates resting on a two-parameter elastic foundation (Pasternak model) using a new theory of high-order shear strain. Duc and Quan [15] conducted the nonlinear stability analysis of double-curved shallow FGM panels on elastic foundations in thermal environments. Kim and his coworkers [16] explored nonlinear vibration and dynamic buckling of eccentrically oblique stiffened FGM plates resting on elastic foundations in a thermal environment. Do et al. [17] presented the role of material combination and new results of mechanical behavior for FG Sandwich plates in a thermal environment. Mai et al. [18] researched the flexural and shear performance of hybrid sandwich panels using an experimental solution. Recently, there have also been a number of publications on mechanical responses of beam and plate structures resting on elastic foundations, in which different foundation models were taken into account. Abdelkrim et al. [19] used the first shear deformation theory to investigate the buckling problem of a single layered graphene sheet resting on elastic foundation. The first shear deformation theory was also used in the work of Noureddine et al. [20] to research the static and free vibration behavior of nanocomposite sandwich plates reinforced by carbon nanotubes resting on Pasternak elastic foundation. Rabhi et al. [21] used a new innovative three unknowns trigonometric shear deformation theory to investigate the buckling and vibration responses of exponentially graded sandwich plates resting on elastic mediums. Fouad et al. [22] used the Navier method and first-order shear deformation beam theory to analyze the dynamic and stability analysis of the simply supported single-walled carbon nanotube-reinforced concrete beam on elastic foundation. Mokhtar et al. [23] investigated the static and free vibration analysis of functionally graded plates based on an efficient and original highorder shear and normal deformation theory. Miloud et al. [24] studied the statics and free vibration of functionally graded porous plates resting on elastic foundations using a new type of quasi-3D hyperbolic shear deformation theory. Farouk et al. [25] researched the effect of Winkler/Pasternak/Kerr foundation and porosity on dynamic behavior of FG plates using a simple quasi-3D hyperbolic theory. Hong [26] investigated the free vibration and static bending analysis of piezoelectric functionally graded material plates resting on one area of the two-parameter elastic foundation using the third-order shear deformation theory of Reddy.

Research on the mechanical behavior of beam and plate structures considering the effect of temperature has also been attracted to scientists and has made some publications. Salah et al. [27] studied the hygrothermal and mechanical buckling responses of simply supported FG sandwich plate seated on Winkler-Pasternak elastic foundation based on a novel shear deformation theory. Abdelouahed et al. [28] used a simple four-variable trigonometric integral shear 
deformation model in order to the static behavior of advanced functionally graded plates subjected to a nonlinear hygro-thermomechanical load. Hakima et al. [29] presented the free vibrational behavior of the FG nanobeams resting on an elastic foundation in the hygrothermal environment. Abderrafik et al. [30] used an exact solution to study the thermomechanical flexural analysis of functionally graded material sandwich plates based on a $n^{\text {th }}$-order shear deformation theory.

Thus, one can see that the above studies have only mentioned the mechanical behaviors of FGM beams and plates without piezoelectric layers, and there is no research on the effects of both elastic foundation and temperature, in which the material properties vary with temperature based on the third-order shear deformation theory. And as we know, FGM structures are often designed to perform in high-temperature environments, and the use of additional piezoelectric layers will increase the control ability and thereby increase the working capacity of these structures; therefore, it is really necessary to study the mechanical behavior of piezoelectric FGM structures, which effectively guide the design and use of these structures in practice. Hence, this work focuses on free and forced vibration of piezoelectric FGM plates resting on elastic foundations, in which the effect of temperature is taken into account, based on the third-order shear deformation theory and the finite element method, which does not need any shear correction factors. This third-order shear deformation theory is suitable for the calculation of plate structures, especially thick thickness plates, which is the outstanding advantage of this theory compared to the classical plate theory and first-order shear deformation theory. This work aims to deal with this problem to figure out the mechanical response of these kinds of structures in practice as well as to contribute the explorations on these issues.

The rest of this paper is organized as follows. Section 2 shortly introduces piezoelectric functionally graded plates. Finite element formulations for piezoelectric FGM plates are described clearly in Section 3. Section 4 is about numerical results and discussion. Some remarkable points are concluded in Section 5.

\section{Piezoelectric Functionally Graded Plates}

Consider a piezoelectric FGM plate, where the core layer is made of functionally graded material, and the two face sheets are actuator and sensor layers. The plate is resting on a twoparameter elastic foundation $k_{w}$ and $k_{s}$, as shown in Figure 1.

Assume that the FGM plate is fabricated from ceramic and metal with mechanical properties varying according to the temperature $\mathrm{T}(\mathrm{K})[31,32]$ :

$$
\Theta=\Theta_{0}\left(\Theta_{-1} T^{-1}+1+\Theta_{1} T^{1}+\Theta_{2} T^{2}+\Theta_{3} T^{3}\right),
$$

in which $\Theta$ represents Young's modulus $E$, Poisson's ratio $v$, and mass density $\rho$, where $T=T_{0}+\Delta T$ and $T_{0}=300 \mathrm{~K}$ (ambient or free stress temperature), $\Delta T$ is the temperature change, and $\Theta_{0}, \Theta_{-1}, \Theta_{1}, \Theta_{2}$, and $\Theta_{3}$ are the temperaturedependent coefficients, which can be seen in Table 1 .

The following equation expresses the dependence of material properties on the volume fraction proportion of ceramic $\left(V_{c}\right)$ and metal $\left(V_{c}\right)$ as follows [31-34]:

$$
\left\{\begin{array}{c}
E(z, T) \\
v(z, T) \\
\rho(z, T)
\end{array}\right\}=\left\{\begin{array}{c}
E_{c}(T) V_{c}+E_{m}(T) V_{m} \\
v_{c}(T) V_{c}+v_{m}(T) V_{m} \\
\rho_{c}(T) V_{c}+\rho_{m}(T) V_{m}
\end{array}\right\},
$$

where $E_{i}, v_{i}, \rho_{i}$, and $V_{i}(i=c, m)$ are Young's modulus, Poisson's ratio, mass density, and the volume fraction of ceramic and metal, respectively. The proportions of ceramic $\left(V_{c}\right)$ and metal $\left(V_{c}\right)$ are changed by the following law [31-35]:

$$
\left\{\begin{array}{l}
V_{c}=\left[1-\left(\frac{z}{h}+\frac{1}{2}\right)^{n}\right], \\
V_{m}=1-V_{c},
\end{array}\right.
$$

in which $n \geq 0$ is the volume fraction index.

\section{Finite Element Formulations for Piezoelectric FGM Plates}

In this work, the higher-order shear deformation theory of Reddy is used; therefore, the displacement field of one point within the plate is expressed as follows [32]:

$$
\left\{\begin{array}{l}
u(x, y, z)=u_{0}(x, y)+z \varphi_{x}(x, y)-\frac{4}{3 h^{2}} z^{3}\left(\varphi_{x}+\frac{\partial w_{0}}{\partial x}\right), \\
v(x, y, z)=v_{0}(x, y)+z \varphi_{y}(x, y)-\frac{4}{3 h^{2}} z^{3}\left(\varphi_{y}+\frac{\partial w_{0}}{\partial y}\right), \\
w(x, y, z)=w_{0}(x, y),
\end{array}\right.
$$

with $u_{0} ; v_{0} ; w_{0}$ are the displacements at a point $(x, y, z=0)$ and $\varphi_{x}$ and $\varphi_{y}$ are the transverse normal rotations in $x z$ and $y z$ surfaces.

The strains can be written in detail as follows:

$$
\left\{\begin{array}{l}
\varepsilon_{x}=\varepsilon_{x}^{m}+z \kappa_{x}^{1}+z^{3} \kappa_{x}^{3} \\
\varepsilon_{y}=\varepsilon_{y}^{m}+z \kappa_{y}^{1}+z^{3} \kappa_{y}^{3} \\
\gamma_{x y}=\gamma_{x y}^{0}+z \kappa_{x y}^{1}+z^{3} \kappa_{x y}^{3} \\
\gamma_{x z}=\gamma_{x z}^{0}+z^{2} \gamma_{x z}^{2} \\
\gamma_{y z}=\gamma_{y z}^{0}+z^{2} \gamma_{y z}^{2}
\end{array}\right.
$$

Equation (5) can be rewritten in the vector form as 


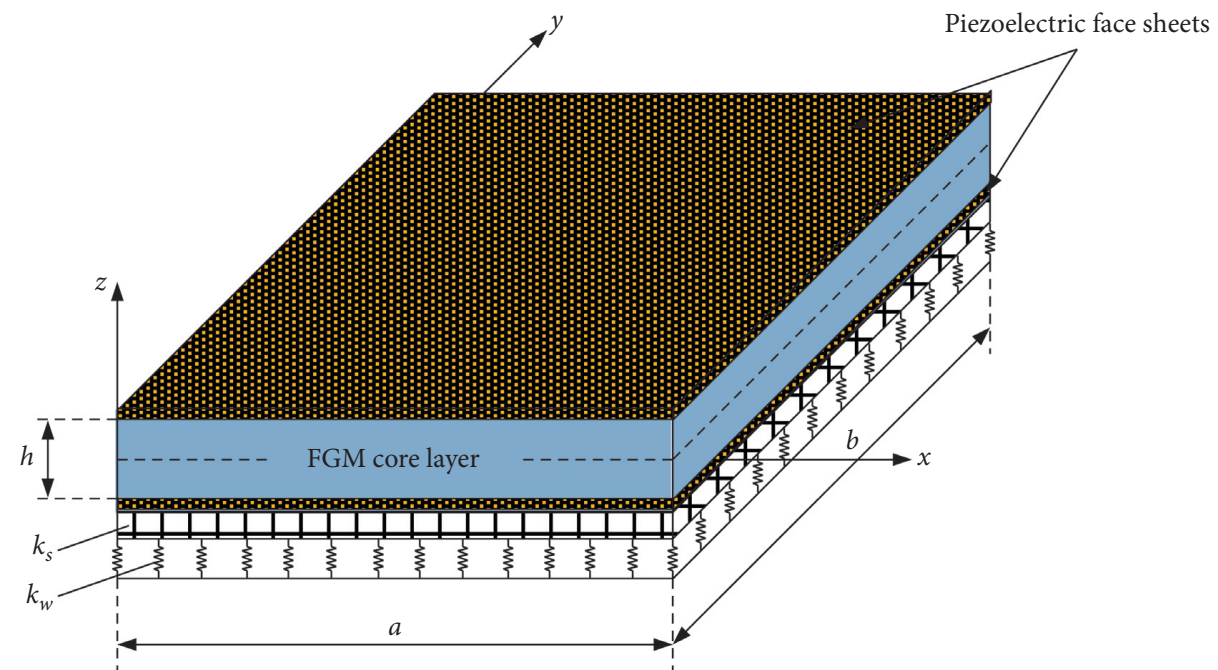

Figure 1: The model of a piezoelectric FGM plate resting on a two-parameter elastic foundation.

TABLE 1: Temperature-dependent coefficient of Young's modulus $E(\mathrm{~Pa})$ and Poisson's ratio $v$ for various materials.

\begin{tabular}{|c|c|c|c|c|c|c|}
\hline Materials & $P_{0}$ & $P_{-1}$ & $P_{1}$ & $P_{2}$ & $P_{3}$ & $P(300 \mathrm{~K})$ \\
\hline \multicolumn{7}{|c|}{ Ceramic silicon nitride $\left(\mathrm{Si}_{3} \mathrm{~N}_{4}\right)$} \\
\hline $\mathrm{E}(\mathrm{Pa})$ & $348.43 \times 10^{9}$ & 0 & $-3.070 \times 10^{-4}$ & $2.160 \times 10^{-7}$ & $-8.946 \times 10^{-11}$ & $322.27 \times 10^{9}$ \\
\hline$v$ & 0.24 & 0 & 0 & 0 & 0 & 0.240 \\
\hline$\rho\left(\mathrm{kg} / \mathrm{m}^{3}\right)$ & 2370 & 0 & 0 & 0 & 0 & 2370 \\
\hline \multicolumn{7}{|c|}{ Ceramic zirconium oxide $\left(\mathrm{ZrO}_{2}\right)$} \\
\hline$E(\mathrm{~Pa})$ & $244.27 \times 10^{9}$ & 0 & $-1.371 \times 10^{-3}$ & $1.214 \times 10^{-6}$ & $-3.681 \times 10^{-10}$ & $168.06 \times 10^{9}$ \\
\hline$v$ & 0.288 & 0 & $1.133 \times 10^{-4}$ & & 0 & 0.298 \\
\hline$\rho\left(\mathrm{kg} / \mathrm{m}^{3}\right)$ & 3657 & 0 & 0 & & 0 & 3657 \\
\hline \multicolumn{7}{|c|}{ Metal stainless steel (SUS304) } \\
\hline$E(\mathrm{~Pa})$ & $201.04 \times 10^{9}$ & 0 & $3.079 \times 10^{-4}$ & $-6.534 \times 10^{-7}$ & 0 & $207.79 \times 10^{9}$ \\
\hline$v$ & 0.326 & 0 & $-2.002 \times 10^{-4}$ & $3.797 \times 10^{-7}$ & 0 & 0.318 \\
\hline$\rho\left(\mathrm{kg} / \mathrm{m}^{3}\right)$ & 8166 & 0 & 0 & 0 & 0 & 8166 \\
\hline
\end{tabular}

$$
\begin{aligned}
& \varepsilon^{m}=\left\{\begin{array}{c}
\varepsilon_{x}^{m} \\
\varepsilon_{y}^{m} \\
\gamma_{x y}^{m}
\end{array}\right\}=\left\{\begin{array}{c}
\frac{\partial u_{0}}{\partial x} \\
\frac{\partial v_{0}}{\partial y} \\
\frac{\partial v_{0}}{\partial x}+\frac{\partial u_{0}}{\partial y}
\end{array}\right\}, \boldsymbol{\kappa}_{x}^{3}=\left\{\begin{array}{c}
\kappa_{x}^{3} \\
\kappa_{y}^{3} \\
\kappa_{x y}^{3}
\end{array}\right\}=\frac{-4}{3 h^{2}}\left\{\begin{array}{c}
\frac{\partial \varphi_{x}}{\partial x}+\frac{\partial^{2} w_{0}}{\partial x^{2}} \\
\frac{\partial \varphi_{y}}{\partial y}+\frac{\partial^{2} w_{0}}{\partial y^{2}} \\
\frac{\partial \varphi_{x}}{\partial y}+\frac{\partial \varphi_{y}}{\partial x}+\frac{2 \partial^{2} w_{0}}{\partial x \partial y}
\end{array}\right\}, \\
& \boldsymbol{\kappa}_{x}^{1}=\left\{\begin{array}{c}
\kappa_{x}^{1} \\
\kappa_{y}^{1} \\
\kappa_{x y}^{1}
\end{array}\right\}=\left\{\begin{array}{c}
\frac{\partial \varphi_{x}}{\partial x} \\
\frac{\partial \varphi_{y}}{\partial y} \\
\frac{\partial \varphi_{x}}{\partial y}+\frac{\partial \varphi_{y}}{\partial x}
\end{array}\right\}, \gamma^{0}=\left\{\begin{array}{c}
\gamma_{x z}^{0} \\
\gamma_{y z}^{0}
\end{array}\right\}=\left\{\begin{array}{c}
\varphi_{x}+\frac{\partial w_{0}}{\partial x} \\
\varphi_{y}+\frac{\partial w_{0}}{\partial y}
\end{array}\right\} ; \\
& \gamma^{2}=\left\{\begin{array}{c}
\gamma_{x z}^{2} \\
\gamma_{y z}^{2}
\end{array}\right\}=\frac{-4}{h^{2}}\left\{\begin{array}{c}
\varphi_{x}+\frac{\partial \omega_{0}}{\partial x} \\
\varphi_{y}+\frac{\partial w_{0}}{\partial y}
\end{array}\right\} .
\end{aligned}
$$


According to Hooke's law, the relation between mechanical components and electrical ones is given by the following expressions $[36,37]$ :

$$
\left\{\begin{array}{l}
\boldsymbol{\sigma}=\mathbf{D}_{b}\left(\boldsymbol{\varepsilon}^{m}+z \boldsymbol{\kappa}^{1}+z^{3} \boldsymbol{\kappa}^{3}\right)-\mathbf{e}^{T} \mathbf{E}_{v} \\
\boldsymbol{\tau}=\mathbf{D}_{s}\left(\boldsymbol{\gamma}^{0}+z^{2} \boldsymbol{\gamma}^{2}\right) \\
\mathbf{D}=\mathbf{e}\left(\boldsymbol{\varepsilon}^{0}+z \boldsymbol{\varepsilon}^{1}+z^{3} \boldsymbol{\varepsilon}^{3}\right)+\mathbf{p} \mathbf{E}_{v}
\end{array}\right.
$$

where

$$
\begin{aligned}
\boldsymbol{\sigma} & =\left\{\begin{array}{c}
\sigma_{x} \\
\sigma_{y} \\
\tau_{x y}
\end{array}\right\}, \\
\boldsymbol{\tau} & =\left\{\begin{array}{c}
\tau_{x z} \\
\tau_{y z}
\end{array}\right\}, \\
\mathbf{D}_{b} & =\frac{E(z, T)}{1-v^{2}(z)}\left[\begin{array}{ccc}
1 & v(z, T) & 0 \\
v(z, T) & 1 & 0 \\
0 & 0 & \frac{1-\nu(z, T)}{2}
\end{array}\right], \\
\mathbf{D}_{s} & =\frac{E(z, T)}{2(1+v(z, T))}\left[\begin{array}{ll}
1 & 0 \\
0 & 1
\end{array}\right] .
\end{aligned}
$$

$\mathbf{E}_{v}$ is the electric field, which is determined through the variation in the potential as follows [38]:

$$
\mathbf{E}_{v}=-\nabla \phi=\left\{\begin{array}{c}
0 \\
0 \\
E^{z}
\end{array}\right\} .
$$

Herein, it is assumed that the electric field applies only to the plate in the thickness direction, and the symbol $E^{z}$ is the electric field in the $z$-direction, which is calculated through the voltage and the thickness of the plate as follows:

$$
E^{z}=\frac{-\phi}{h_{p}} .
$$

D is the electric displacement vector and e denotes the piezoelectric stress coefficients, which has a specific expression in the following form:

$$
\mathbf{e}=\left[\begin{array}{ccc}
0 & 0 & 0 \\
0 & 0 & 0 \\
e_{31} & e_{32} & 0
\end{array}\right]
$$

$\mathbf{p}$ is the dielectric tensor at constant mechanical strain with the expression as follows:

$$
\mathbf{p}=\left[\begin{array}{ccc}
p_{11} & 0 & 0 \\
0 & p_{22} & 0 \\
0 & 0 & p_{33}
\end{array}\right] .
$$

This work uses an 8-node plate element, each node has 5 degrees of freedom, and displacement components at the neutral plane are interpolated as follows:

$$
\begin{aligned}
& u_{0}=\sum_{j=1}^{8} N_{j}(\xi, \eta) \cdot u_{j}, \\
& v_{0}=\sum_{j=1}^{8} N_{j}(\xi, \eta) \cdot v_{j}, \\
& w_{0}=\sum_{j=1}^{8} N_{j}(\xi, \eta) \cdot w_{0 j}, \\
& \varphi_{x}=\sum_{j=1}^{8} N_{j}(\xi, \eta) \cdot \varphi_{x j}, \\
& \varphi_{y}=\sum_{j=1}^{8} N_{j}(\xi, \eta) \cdot \varphi_{y j},
\end{aligned}
$$

where $N_{j}$ is the Lagrangian interpolation function of the 8node plate element and $\xi$ and $\eta$ are natural coordinates.

Based on the above interpolation technique, the strain components are now written as follows:

$$
\left\{\begin{array}{l}
\varepsilon^{m}=\mathbf{B}_{0} \mathbf{q}_{e} ; \boldsymbol{\kappa}^{1}=\mathbf{B}_{1} \mathbf{q}_{e} ; \boldsymbol{\kappa}^{3}=\mathbf{B}_{3} \mathbf{q}_{e}, \\
\boldsymbol{\gamma}^{0}=\mathbf{B}_{0 \gamma} \mathbf{q}_{e} ; \gamma^{2}=\mathbf{B}_{2 \gamma} \mathbf{q}_{e},
\end{array}\right.
$$

with

$$
\begin{gathered}
\mathbf{B}_{0}=\sum_{j=1}^{8}\left[\begin{array}{ccccc}
\frac{\partial N_{j}}{\partial x} & 0 & 0 & 0 & 0 \\
0 & \frac{\partial N_{j}}{\partial y} & 0 & 0 & 0 \\
\frac{\partial N_{j}}{\partial y} & \frac{\partial N_{j}}{\partial x} & 0 & 0 & 0
\end{array}\right], \\
\mathbf{B}_{1}=\sum_{j=1}^{8}\left[\begin{array}{lllll}
0 & 0 & 0 & \frac{\partial N_{j}}{\partial x} & 0 \\
0 & 0 & 0 & 0 & \frac{\partial N_{j}}{\partial y} \\
0 & 0 & 0 & \frac{\partial N_{j}}{\partial y} & \frac{\partial N_{j}}{\partial x}
\end{array}\right],
\end{gathered}
$$




$$
\begin{aligned}
& \mathbf{B}_{3}=\frac{-4}{3 h^{2}} \sum_{j=1}^{8}\left[\begin{array}{ccccc}
0 & 0 & \frac{\partial^{2} N_{j}}{\partial x^{2}} & \frac{\partial N_{j}}{\partial x} & 0 \\
0 & 0 & \frac{\partial^{2} N_{j}}{\partial y^{2}} & 0 & \frac{\partial N_{j}}{\partial y} \\
0 & 0 & 2 \frac{\partial^{2} N_{j}}{\partial x \partial y} & \frac{\partial N_{j}}{\partial y} & \frac{\partial N_{j}}{\partial x}
\end{array}\right], \\
& \mathbf{B}_{0 \gamma}=\sum_{j=1}^{8}\left[\begin{array}{ccccc}
0 & 0 & \frac{\partial N_{j}}{\partial x} & 0 & N_{j} \\
0 & 0 & \frac{\partial N_{j}}{\partial y} & N_{j} & 0
\end{array}\right], \\
& \mathbf{B}_{2 \gamma}=\frac{-4}{h^{2}} \sum_{j=1}^{8}\left[\begin{array}{ccccc}
0 & 0 & \frac{\partial N_{j}}{\partial x} & 0 & N_{j} \\
0 & 0 & \frac{\partial N_{j}}{\partial y} & N_{j} & 0
\end{array}\right] \text {. }
\end{aligned}
$$

At this point, the electric field vector of the actuator layer and the sensor layer is calculated through two degrees of freedom $\phi_{a}$ and $\phi_{s}$ as follows:

$$
\mathbf{E}_{v}=-\left[\begin{array}{c}
0,0, \frac{1}{h_{a}} \\
0,0, \frac{1}{h_{s}}
\end{array}\right]^{T}\left\{\begin{array}{l}
\phi_{a} \\
\phi_{s}
\end{array}\right\}=-\left[\begin{array}{c}
\mathbf{B}_{\phi}^{a} \\
\mathbf{B}_{\phi}^{s}
\end{array}\right]\left\{\begin{array}{l}
\phi_{a} \\
\phi_{s}
\end{array}\right\},
$$

where

$$
\begin{aligned}
& \mathbf{B}_{\phi}^{a}=\left[0,0, \frac{1}{h_{a}}\right]^{T}, \\
& \mathbf{B}_{\phi}^{s}=\left[0,0, \frac{1}{h_{s}}\right]^{T},
\end{aligned}
$$

in which $h_{a}$ and $h_{s}$ are the thicknesses of the actuator layer and sensor layer, respectively.

The internal force components can be expressed as

$$
\left\{\begin{array}{c}
\mathbf{N} \\
\mathbf{M} \\
\widehat{M} \\
\mathbf{Q} \\
\bar{Q}
\end{array}\right\}=\left[\begin{array}{ccccc}
\mathbf{A} & \mathbf{B} & \mathbf{E} & 0 & 0 \\
\mathbf{B} & \mathbf{D} & \mathbf{F} & 0 & 0 \\
\mathbf{E} & \mathbf{F} & \mathbf{H} & 0 & 0 \\
0 & 0 & 0 & \bar{A} & \bar{B} \\
0 & 0 & 0 & \bar{B} & \bar{D}
\end{array}\right]\left\{\begin{array}{c}
\boldsymbol{\varepsilon}^{m} \\
\boldsymbol{\kappa}^{1} \\
\boldsymbol{\kappa}^{3} \\
\boldsymbol{\gamma}^{0} \\
\boldsymbol{\gamma}^{2}
\end{array}\right\},
$$

where

$$
\begin{aligned}
& \left\{\begin{array}{l}
\mathbf{A} \\
\mathbf{B} \\
\mathbf{D} \\
\mathbf{E} \\
\mathbf{F} \\
\mathbf{H}
\end{array}\right\}=\int_{-h / 2}^{h / 2} \mathbf{D}_{b}\left\{\begin{array}{c}
1 \\
\mathbf{z} \\
\mathbf{z}^{2} \\
\mathbf{z}^{3} \\
\mathbf{z}^{4} \\
\mathbf{z}^{6}
\end{array}\right\} \mathrm{d} \mathbf{z}, \\
& \left\{\begin{array}{c}
\bar{A} \\
\bar{B} \\
\bar{D}
\end{array}\right\}=\int_{-h / 2}^{h / 2} \mathbf{D}_{s}\left\{\begin{array}{c}
1 \\
\mathbf{z}^{2} \\
\mathbf{z}^{4}
\end{array}\right\} \mathrm{d} \mathbf{z} .
\end{aligned}
$$

The total strain energy of the piezoelectric FGM plate can be given by 


$$
\begin{aligned}
& \prod_{e}=\frac{1}{2} \int_{S_{e}}\left(\begin{array}{c}
\left\{\begin{array}{ccc}
\boldsymbol{\varepsilon}^{0} & \boldsymbol{\varepsilon}^{1} & \boldsymbol{\varepsilon}^{3}
\end{array}\right\}^{T} \boldsymbol{\sigma}+\left\{\begin{array}{cc}
\boldsymbol{\gamma}^{0} & \boldsymbol{\gamma}^{2}
\end{array}\right\}^{T} \boldsymbol{\tau}-\mathbf{D}^{T} \mathbf{E}_{v} \\
k_{w} w^{2}+k_{s}\left(\left(\frac{\partial w}{\partial x}\right)^{T}\left(\frac{\partial w}{\partial x}\right)+\left(\frac{\partial w}{\partial y}\right)^{T}\left(\frac{\partial w}{\partial y}\right)\right)
\end{array}\right) \mathrm{d} S \mathrm{~d} z-\int_{S_{e}} \mathbf{u}^{T} \widehat{F} \mathrm{~d} S-\int_{S_{e}} \mathbf{E}_{v}^{T} \overline{\mathbf{Q}} \mathrm{d} S, \\
& =\mathbf{q}_{e}^{T} \frac{1}{2} \int_{S_{e}}\left(\begin{array}{c}
\mathbf{B}_{0}^{T} \mathbf{A} \mathbf{B}_{0}+\mathbf{B}_{0}^{T} \mathbf{B B}_{1}+\mathbf{B}_{1}^{T} \mathbf{E B}_{3}+\mathbf{B}_{1}^{T} \mathbf{B B}_{0}+\mathbf{B}_{1}^{T} \mathbf{D} \mathbf{B}_{1}+\mathbf{B}_{0}^{T} \mathbf{E B}_{3} \\
+\mathbf{B}_{3}^{T} \mathbf{E B}_{0}+\mathbf{B}_{1}^{T} \mathbf{F} \mathbf{B}_{3}+\mathbf{B}_{3}^{T} \mathbf{E B}_{1}+\mathbf{B}_{3}^{T} \mathbf{F} \mathbf{B}_{1}+\mathbf{B}_{3}^{T} \mathbf{H} \mathbf{B}_{3} \\
+\mathbf{B}_{0 \gamma}^{T} \overline{\mathbf{A}} \mathbf{B}_{0 \gamma}+\mathbf{B}_{0 \gamma}^{T} \overline{\mathbf{B}} \mathbf{B}_{2 \gamma}+\mathbf{B}_{2 \gamma}^{T} \overline{\mathbf{B}} \mathbf{B}_{0 \gamma}+\mathbf{B}_{2 \gamma}^{T} \overline{\mathbf{B}} \mathbf{B}_{2 \gamma} \\
+k_{w} \mathbf{N}_{w}^{T} \mathbf{N}_{w}+k_{s}\left(\left(\frac{\partial \mathbf{N}_{w}}{\partial x}\right)^{T}\left(\frac{\partial \mathbf{N}_{w}}{\partial x}\right)+\left(\frac{\partial \mathbf{N}_{w}}{\partial y}\right)^{T}\left(\frac{\partial \mathbf{N}_{w}}{\partial y}\right)\right)
\end{array}\right) d S \mathbf{q}_{e} \\
& -\mathbf{q}_{e}^{T} \frac{1}{2} \int_{S_{e}}\left(-\mathbf{B}_{0}^{T} \mathbf{A} \mathbf{e}^{T} \mathbf{B}_{\phi a}-\mathbf{B}_{1}^{T} \mathbf{B e}^{T} \mathbf{B}_{\phi a}-\mathbf{B}_{3}^{T} \mathbf{E e}^{T} \mathbf{B}_{\phi a}\right) \mathrm{d} S \phi_{e a} \\
& -\mathbf{q}_{e}^{T} \frac{1}{2} \int_{S_{e}}\left(-\mathbf{B}_{0}^{T} \mathbf{A} \mathbf{e}^{T} \mathbf{B}_{\phi s}-\mathbf{B}_{1}^{T} \mathbf{B e}^{T} \mathbf{B}_{\phi s}-\mathbf{B}_{3}^{T} \mathbf{E e}^{T} \mathbf{B}_{\phi s}\right) \mathrm{d} S \phi_{e s} \\
& -\phi_{e a}^{T} \frac{1}{2} \int_{S_{e}}\left(-\mathbf{B}_{\phi a}^{T} \mathbf{e} \mathbf{A} \mathbf{B}_{0}-\mathbf{B}_{\phi a}^{T} \mathbf{e} \mathbf{B} \mathbf{B}_{1}-\mathbf{B}_{\phi a}^{T} \mathbf{e E B _ { 3 }}\right) \mathrm{d} S \mathbf{q}_{e} \\
& -\phi_{e s}^{T} \frac{1}{2} \int_{S_{e}}\left(-\mathbf{B}_{\phi s}^{T} \mathbf{e} \mathbf{A} \mathbf{B}_{0}-\mathbf{B}_{\phi s}^{T} \mathbf{e B B}_{1}-\mathbf{B}_{\phi s}^{T} \mathbf{e E B _ { 3 }}\right) \mathrm{d} S \mathbf{q}_{e} \\
& -\frac{1}{2} \phi_{e a}^{T} \int_{S_{e}} \mathbf{B}_{\phi a}^{T} \mathbf{p} \mathbf{B}_{\phi a}^{T} \mathrm{~d} S \phi_{e a}-\frac{1}{2} \phi_{e s}^{T} \int_{S_{e}} \mathbf{B}_{\phi s}^{T} \mathbf{p} \mathbf{B}_{\phi s}^{T} \mathrm{~d} S \phi_{e s}-\mathbf{q}_{e}^{T} \int_{S_{e}} \mathbf{N}^{T} \widehat{F} \mathrm{~d} S \\
& -\phi_{a e}^{T} \int_{S_{e}} \mathbf{B}_{\phi}^{a T} \bar{Q}^{a} \mathrm{~d} S-\phi_{s e}^{T} \int_{S_{e}} \mathbf{B}_{\phi}^{s T} \bar{Q}^{s} \mathrm{~d} S,
\end{aligned}
$$

where $\mathbf{N}_{w}=\sum_{j=1}^{8}\left[0,0, N_{j}, 0,0\right]$ and $\widehat{F}$ is the surface loading and equation (20) is expressed in matrix form as follows:

$$
\prod_{e}=\frac{1}{2}\left(\begin{array}{c}
\mathbf{q}_{e}^{T} \mathbf{K}_{u u e} \mathbf{q}_{e}+\mathbf{q}_{e}^{T} \mathbf{K}_{u \phi e}^{a} \phi_{a e}+\mathbf{q}_{e}^{T} \mathbf{K}_{u \phi e}^{s} \phi_{s e} \\
+\phi_{a e}^{T} \mathbf{K}_{\phi u e}^{a} \mathbf{q}_{e}+\phi_{s e}^{T} \mathbf{K}_{\phi u e}^{s} q_{e} \\
-\phi_{a e}^{T} \mathbf{K}_{\phi \phi e}^{a} \phi_{a e}-\phi_{s e}^{T} \mathbf{K}_{\phi \phi e}^{s} \phi_{s e}
\end{array}\right)-\mathbf{q}_{e}^{T} \mathbf{P}_{e}-\phi_{a e}^{T} \mathbf{F}_{q e}^{a}-\phi_{s e}^{T} \mathbf{F}_{q e}^{s},
$$


where each matrix is defined clearly as

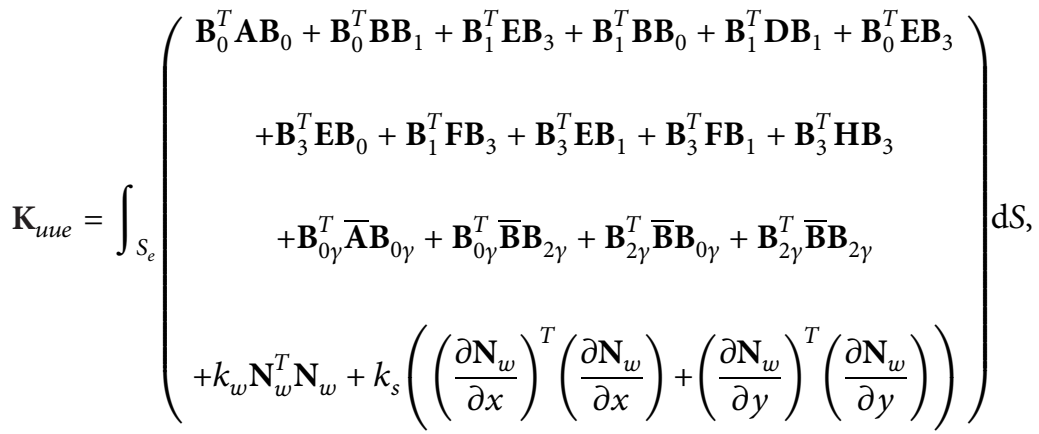

$$
\begin{aligned}
& \mathbf{K}_{u \phi e}^{a}=\int_{S_{e}}\left(-\mathbf{B}_{0}^{T} \mathbf{A} \mathbf{e}^{T} \mathbf{B}_{\phi a}-\mathbf{B}_{1}^{T} \mathbf{B} \mathbf{e}^{T} \mathbf{B}_{\phi a}-\mathbf{B}_{3}^{T} \mathbf{E e}^{T} \mathbf{B}_{\phi a}\right) \mathrm{d} S, \\
& \mathbf{K}_{u \phi e}^{s}=\int_{S_{e}}\left(-\mathbf{B}_{0}^{T} \mathbf{A} \mathbf{e}^{T} \mathbf{B}_{\phi s}-\mathbf{B}_{1}^{T} \mathbf{B e}^{T} \mathbf{B}_{\phi s}-\mathbf{B}_{3}^{T} \mathbf{E e}^{T} \mathbf{B}_{\phi s}\right) \mathrm{d} S, \\
& \mathbf{K}_{\phi u e}^{a}=\int_{S_{e}}\left(-\mathbf{B}_{\phi a}^{T} \mathbf{e} \mathbf{A B}_{0}-\mathbf{B}_{\phi a}^{T} \mathbf{e} \mathbf{B} \mathbf{B}_{1}-\mathbf{B}_{\phi a}^{T} \mathbf{e E B} \mathbf{B}_{3}\right) \mathrm{d} S, \\
& \mathbf{K}_{\phi u e}^{s}=\int_{S_{e}}\left(-\mathbf{B}_{\phi s}^{T} \mathbf{e} \mathbf{A} \mathbf{B}_{0}-\mathbf{B}_{\phi s}^{T} \mathbf{e B B} \mathbf{B}_{1}-\mathbf{B}_{\phi s}^{T} \mathbf{e E B _ { 3 }}\right) \mathrm{d} S, \\
& \mathbf{K}_{\phi \phi e}^{a}=\phi_{e a}^{T} \int_{S_{e}} \mathbf{B}_{\phi a}^{T} \mathbf{p} \mathbf{B}_{\phi a}^{T} \mathrm{~d} S \phi_{e a}, \\
& \mathbf{K}_{\phi \phi e}^{s}=\phi_{e s}^{T} \int_{S_{e}} \mathbf{B}_{\phi s}^{T} \mathbf{p} \mathbf{B}_{\phi s}^{T} \mathrm{~d} S \phi_{e s}, \\
& \mathbf{P}_{e}=\int_{S_{e}} \mathbf{N}^{T} \widehat{F} \mathrm{~d} S_{e}, \\
& \mathbf{F}_{q e}^{a}=\int_{S_{e}} \mathbf{B}_{\phi}^{a T} \mathbf{Q}^{a} \mathrm{~d} S \\
& \mathbf{F}_{q e}^{s}=\int_{S_{e}} \mathbf{B}_{\phi}^{s T} \mathbf{Q}^{s} \mathrm{~d} S,
\end{aligned}
$$

in which $Q^{a}$ and $Q^{s}$ are the voltages acting on actuator and sensor layers.
The kinetic energy of the plate element is determined by the following formula:

$$
\begin{aligned}
T_{e} & =\frac{1}{2} \int_{V_{e}} \dot{u}^{T} \rho(x, z) \dot{u} \mathrm{~d} V=\frac{1}{2} \dot{q}_{e}^{T}\left(\begin{array}{c}
\int_{V_{e}} \mathbf{N}^{T} \mathbf{L}^{T} \rho_{p}(x) \mathbf{L N} \mathrm{d} V+\int_{V_{e}} \mathbf{N}^{T} \mathbf{L}^{T} \rho_{a}(x) \mathbf{L N} \mathrm{d} V \\
\int_{V_{e}} \mathbf{N}^{T} \mathbf{L}^{T} \rho_{s}(x) \mathbf{L N} \mathrm{N} V
\end{array}\right) \dot{q}_{e} \\
& =\frac{1}{2} \dot{q}_{e}^{T} \mathbf{M}_{e} \dot{q}_{e},
\end{aligned}
$$


where $\rho_{p}, \rho_{a}$, and $\rho_{s}$ are, respectively, the mass density of the plate, actuator, and sensor layers; $N$ is the shape function matrix; and the matrix $\boldsymbol{L}$ and the element mass matrix $\boldsymbol{M}_{e}$ are defined as follows:

$$
\begin{aligned}
\mathbf{L} & =\left[\begin{array}{ccccc}
1 & 0 & -\frac{4 z^{3}}{3 h^{2}} \frac{\partial}{\partial x} z-\frac{4 z^{3}}{3 h^{2}} & 0 \\
0 & 1 & -\frac{4 z^{3}}{3 h^{2}} \frac{\partial}{\partial y} & 0 & z-\frac{4 z^{3}}{3 h^{2}} \\
0 & 0 & 1 & 0 & 0
\end{array}\right], \\
\mathbf{M}_{e} & =\left(\begin{array}{c}
\int_{V_{e}} \mathbf{N}^{T} \mathbf{L}^{T} \rho_{p}(x) \mathbf{L} \mathbf{N} \mathrm{d} V+\int_{V_{e}} \mathbf{N}^{T} \mathbf{L}^{T} \rho_{a}(x) \mathbf{L N} \mathrm{N} V \\
\int_{V_{e}} \mathbf{N}^{T} \mathbf{L}^{T} \rho_{s}(x) \mathbf{L N} \mathrm{N} V
\end{array}\right) .
\end{aligned}
$$

The Galerkin weak form of the governing equations of piezoelectric plates can be derived by using Hamilton's variational principle which can be written as follows:

$$
\delta L_{e}=0 \text {, }
$$

where

$$
L_{e}=T_{e}-\Pi_{e} .
$$

Substituting equations (21) and (23) into equation (25), the following equation can be obtained:

$$
\begin{aligned}
& {\left[\begin{array}{ccc}
\mathbf{M}_{u e} & 0 & 0 \\
0 & 0 & 0 \\
0 & 0 & 0
\end{array}\right]\left\{\ddot{q}_{e} \ddot{\phi}_{a e} \ddot{\phi}_{s e}\right\}+\left[\begin{array}{ccc}
\mathbf{K}_{u u e} & \mathbf{K}_{u \phi e}^{a} & \mathbf{K}_{u \phi e}^{s} \\
\mathbf{K}_{\phi u e}^{a} & \mathbf{K}_{\phi \phi e}^{a} & 0 \\
\mathbf{K}_{\phi u e}^{s} & 0 & \mathbf{K}_{\phi \phi e}^{s}
\end{array}\right]\left\{\begin{array}{c}
\mathbf{q}_{e} \\
\phi_{a e} \\
\phi_{s e}
\end{array}\right\}} \\
& \quad=\left\{\begin{array}{c}
\mathbf{P}_{e} \\
\mathbf{F}_{q e}^{a} \\
\mathbf{F}_{q e}^{s}
\end{array}\right\} .
\end{aligned}
$$

By extracting $\phi_{a e}$ and $\phi_{s e}$ from the second and third expressions of equation (27) and then substituting them into the first expression of equation (27), the forced vibration equation of the piezoelectric plate element is obtained as follows:

$$
\mathbf{M}_{u e} \ddot{q}_{e}+\left(\begin{array}{c}
\mathbf{K}_{u u e}+\mathbf{K}_{u \phi e}^{a} \mathbf{K}_{\phi \phi e}^{a-1} \mathbf{K}_{\phi u e}^{a} \\
+\mathbf{K}_{u \phi e}^{s} \mathbf{K}_{\phi \phi e}^{s-1} \mathbf{K}_{\phi u e}^{s}
\end{array}\right) \mathbf{q}_{e}=\mathbf{P}_{e}+\mathbf{K}_{u \phi e}^{s} \mathbf{F}_{q e}^{s}+\mathbf{K}_{u \phi e}^{a} \mathbf{F}_{q e}^{a} .
$$

As external forces are acting to the plate, the plate will be deformed, and the voltage will be changed; then, the voltage of the sensor layer will be transmitted to the controller, and the controller will overpower the applied voltage back to the actuator layer to create an electrical force that acts opposite to the direction in which the plate is deforming, returning the plate to its equilibrium before deformation. The process is clearly described in Figure 2 .

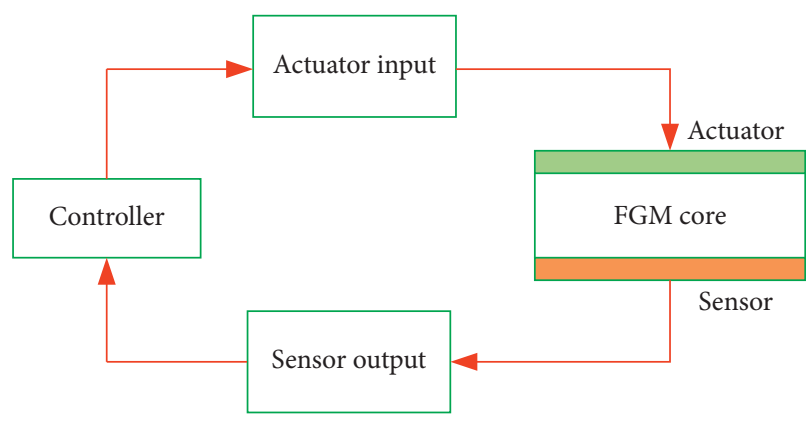

Figure 2: The vibration control model of the plate by the piezoelectric layers.

Let $G_{d}$ be the displacement feedback coefficient and $G_{v}$ the velocity feedback coefficient of the controller, and these coefficients present the relationship between the applied voltage (input) $\phi_{a}$ and the voltage in the sensor layer (output) $\phi_{s}$ :

$$
\phi_{a e}=G_{d} \phi_{s e}+G_{v} \dot{\phi}_{s e} .
$$

Without the appearance of the applied voltages $\mathbf{Q}^{a}$ and $\mathbf{Q}^{s}$, from the third equation in (27), the voltage vector of the sensor layer is derived as follows:

$$
\phi_{s e}=\mathbf{K}_{\phi \phi}^{s-1} \mathbf{K}_{u \phi}^{s} \mathbf{q}_{e},
$$

and the voltage of the sensor layer is generated by the deformation phenomenon of the plate as follows:

$$
\mathbf{Q}_{e}^{s}=\mathbf{K}_{u \phi e}^{s} \mathbf{q}_{e} .
$$

This means that when the plate is oscillating, the voltage generated in the sensor layer due to the deformation effect of the plate will generate an input signal to the controller, which will affect directly the actuator layer. As a result, the stress and strain generated from the piezoelectric effect will produce a voltage signal applied back to it, controlling the stress and strain in the desired direction.

Substituting expressions (29) and (30) into the second equation in (27), we obtain the following expression:

$$
\mathbf{Q}_{e}^{a}=\mathbf{K}_{u \phi e}^{a} \mathbf{q}_{e}-\mathbf{G}_{d} \mathbf{K}_{u \phi}^{a} \mathbf{K}_{\phi \phi e}^{s-1} \mathbf{K}_{\phi u e}^{s} \mathbf{q}_{e}-\mathbf{G}_{v} \mathbf{K}_{u \phi e}^{a} \mathbf{K}_{\phi \phi e}^{s-1} \mathbf{K}_{\phi u e}^{s} \dot{q}_{e} .
$$

Substituting equation (32) into (28), we get the forced vibration equation of the piezoelectric element as follows:

$$
\mathbf{M}_{u e} \ddot{q}_{e}+\mathbf{C}_{e}^{d} \dot{q}_{e}+\mathbf{K}_{e}^{*} \mathbf{q}_{e}=\mathbf{P}_{e},
$$

where

$$
\mathbf{K}_{e}^{*}=\mathbf{K}_{u u e}+G_{d} \mathbf{K}_{u \phi e}^{a} \mathbf{K}_{\phi \phi e}^{s-1} \mathbf{K}_{\phi u e}^{s},
$$

and $\mathbf{C}_{e}^{d}$ is the resistance matrix due to the effect of the feedback coefficient which is determined by the following expression:

$$
\mathbf{C}_{e}^{d}=G_{v} \mathbf{K}_{u \phi e}^{a} \mathbf{K}_{\phi \phi e}^{s-1} \mathbf{K}_{\phi u e}^{s} .
$$


TABLE 2: Comparison of first natural frequencies $(\mathrm{Hz})$ with some meshes.

\begin{tabular}{cccccccccc}
\hline \multirow{2}{*}{ Mode } & \multirow{2}{*}{ Method } & Mesh & & & & \multicolumn{3}{c}{$n$} & \\
& & & 0 & 0.2 & 0.5 & 1 & 5 & 1000 \\
\hline \multirow{4}{*}{$139]$} & - & 144.2 & 168.7 & 185.4 & 198.9 & 230.4 & 247.3 & 261.7 \\
1 & {$[40]$} & - & 145.3 & 169.2 & 186.2 & 200.5 & 233.0 & 250.3 & 265.1 \\
& & $4 \times 4$ & 146.1 & 170.6 & 175.5 & 201.7 & 233.8 & 250.7 & 265.1 \\
& & $6 \times 6$ & 145.4 & 169.8 & 186.6 & 200.7 & 232.5 & 249.4 & 263.8 \\
& \multirow{4}{*}{ This work } & $\mathbf{8} \times \mathbf{8}$ & $\mathbf{1 4 5 . 4}$ & $\mathbf{1 6 9 . 8}$ & $\mathbf{1 8 6 . 6}$ & $\mathbf{2 0 0 . 6}$ & $\mathbf{2 3 2 . 4}$ & $\mathbf{2 4 9 . 3}$ & $\mathbf{2 6 3 . 7}$ \\
& & $10 \times 10$ & 145.4 & 169.8 & 186.6 & 200.6 & 232.4 & 249.3 & 263.7 \\
& & $12 \times 12$ & 145.4 & 169.8 & 186.6 & 200.6 & 232.4 & 249.3 & 263.7 \\
\hline
\end{tabular}

TABLE 3: Comparison of first nondimensional natural frequencies $@$ of fully simply supported FGM plates resting on two-parameter elastic foundation.

\begin{tabular}{|c|c|c|c|c|}
\hline$\left(k_{w}^{*}, k_{s}^{*}\right)$ & $a / h$ & $N$ & [41] & This work \\
\hline \multirow{10}{*}{$(0,0)$} & \multirow{6}{*}{20} & 0 & 0.0239 & 0.0239 \\
\hline & & 0.25 & 0.0226 & 0.0231 \\
\hline & & 1 & 0.0215 & 0.0220 \\
\hline & & 5 & 0.0218 & 0.0225 \\
\hline & & $\infty$ & 0.0204 & 0.0206 \\
\hline & & 0 & 0.0918 & 0.0919 \\
\hline & \multirow{4}{*}{10} & 0.25 & 0.0860 & 0.0888 \\
\hline & & 1 & 0.0815 & 0.0848 \\
\hline & & 5 & 0.0817 & 0.0860 \\
\hline & & $\infty$ & 0.0789 & 0.0790 \\
\hline \multirow{10}{*}{$(250,25)$} & \multirow{6}{*}{20} & 0 & 0.0342 & 0.0342 \\
\hline & & 0.25 & 0.0328 & 0.0331 \\
\hline & & 1 & 0.0318 & 0.0321 \\
\hline & & 5 & 0.0323 & 0.0328 \\
\hline & & $\infty$ & 0.0293 & 0.0294 \\
\hline & & 0 & 0.1336 & 0.1337 \\
\hline & \multirow{4}{*}{10} & 0.25 & 0.1277 & 0.1295 \\
\hline & & 1 & 0.1238 & 0.1258 \\
\hline & & 5 & 0.1253 & 0.1279 \\
\hline & & $\infty$ & 0.1148 & 0.1148 \\
\hline
\end{tabular}

In the case of taking into account the structural damping coefficient, the forced vibration equation of piezoelectric plate element has the following form:

$$
\mathbf{M}_{u e} \ddot{q}_{e}+\left(\mathbf{C}_{e}^{d}+\mathbf{C}_{e}\right) \dot{q}_{e}+\mathbf{K}_{e}^{*} \mathbf{q}_{e}=\mathbf{P}_{e}
$$

where $\mathbf{C}_{e}$ is the Rayleigh structural damping matrix, which is calculated through the mass and stiffness matrices as follows:

$$
\mathbf{C}_{e}=\bar{\alpha} \mathbf{M}_{u u e}+\bar{\beta} \mathbf{K}_{u u e} .
$$

After assembling the element matrices and eliminating boundary conditions, the forced vibration equation of the piezoelectric plate is expressed as follows:

$$
\mathbf{M}_{u} \ddot{q}+\left(\mathbf{C}^{d}+\mathbf{C}\right) \dot{q}+\mathbf{K}^{*} \mathbf{q}=\breve{P} .
$$

For the free vibration problem, the following equation is established to find natural frequencies and vibration mode shapes:

$$
\left(\mathbf{K}^{*}-\omega^{2} \mathbf{M}_{u}\right) \mathbf{q}=0
$$

Thus, finite element formulations established in this work used the third-order shear deformation theory, which does not need any shear correction factors, and this is the outstanding advantage in comparison with the first-order shear deformation theory and classical plate theory. At the same time, this theory also considers the effect of shear strain, so it will correctly show the mechanical response of the plates, especially the plate structures with large thickness.

\section{Numerical Results}

\subsection{Free Vibration of a Piezoelectric FGM Plate}

4.1.1. Accuracy Study. This section firstly carries the verification problem to show the accuracy of the proposed theory and mathematical model. The results of this work are compared with those of references [39, 40], where analytical and numerical methods were used. Consider a piezoelectric FGM (Ti-6Al-4 V/aluminum oxide) with dimensions $a=b=0.4 \mathrm{~m}$ and thickness $h_{p}=5 \mathrm{~mm}$. Material properties are $E_{c}=320.24 \mathrm{GPa}, v_{c}=0.26, \rho_{c}=3750 \mathrm{~kg} /$ $\mathrm{m}^{3}, E_{m}=105.7 \mathrm{GPa}, v_{m}=0.2981$, and $\rho_{m}=4429 \mathrm{~kg} / \mathrm{m}^{3}$. Two piezoelectric face sheets have thicknesses $h_{a}=h_{s}=0.1 \mathrm{~mm}$, and material properties Epi $=63 \mathrm{GPa}$, $v_{p i}=0.3, d_{31}=d_{32}=254.10^{-12} \mathrm{~m} / \mathrm{V}$, and $k_{33}=15 \mathrm{nF} / \mathrm{m}$. The plate is fully simply supported (SSSS). Table 2 presents the numerical results with some meshes, and it can be seen 
TABLE 4: Nondimensional natural frequencies $\left(\bar{\Psi}=\left(\omega a^{2} / h\right)\left[\rho_{0}\left(1-v^{2}\right) / E_{0}\right]^{1 / 2}\right)$ of $\mathrm{Si}_{3} \mathrm{~N}_{4} / \mathrm{SUS} 04$ plates $\left(E_{0}\right.$ and $\rho_{0}$ are the reference values of $E_{m}$ and $\rho_{m}$ at $\left.T_{0}=300 \mathrm{~K}\right)$.

\begin{tabular}{|c|c|c|c|c|c|c|c|c|c|}
\hline \multirow{2}{*}{$T(\mathrm{~K})$} & \multicolumn{3}{|c|}{$n=0.5$} & \multicolumn{3}{|c|}{$n=1$} & \multicolumn{3}{|c|}{$n=5$} \\
\hline & [42] & [31] & This work & [42] & [31] & This work & [42] & [31] & This work \\
\hline 400 & 15.938 & 15.355 & 15.467 & 13.915 & 13.433 & 13.646 & 11.175 & 10.852 & 11.012 \\
\hline 500 & 15.468 & 15.192 & 15.285 & 13.462 & 13.280 & 13.332 & 10.749 & 10.716 & 10.723 \\
\hline 600 & 14.939 & 15.008 & 14.998 & 12.941 & 13.093 & 13.001 & 10.242 & 10.528 & 10.210 \\
\hline
\end{tabular}

TABLE 5: First nondimensional natural frequencies $₫$ of $\mathrm{Si}_{3} \mathrm{~N}_{4} /$ SUS304 plates depending on the temperature $\left(k_{w}^{*}, k_{s}^{*}\right)=(100,10)$.

\begin{tabular}{lcccc}
\hline$n$ & \multicolumn{3}{c}{$T(\mathrm{~K})$} \\
& 300 & 400 & 500 & 600 \\
\hline 0 & 0.0363 & 0.0360 & 0.0357 & 0.0354 \\
0.5 & 0.0287 & 0.0284 & 0.0282 & 0.0279 \\
1 & 0.0262 & 0.0260 & 0.0258 & 0.0255 \\
2 & 0.0243 & 0.0241 & 0.0239 & 0.0236 \\
10 & 0.0220 & 0.0218 & 0.0216 & 0.0214 \\
\hline
\end{tabular}

TABLE 6: First nondimensional natural frequencies $₫$ of $\mathrm{ZrO}_{2} /$ SUS304 plates depending on the temperature $\left(k_{w}^{*}, k_{s}^{*}\right)=(100,10)$.

\begin{tabular}{lcccc}
\hline$N$ & \multicolumn{3}{c}{$T(\mathrm{~K})$} \\
& 300 & 400 & 500 & 600 \\
\hline 0 & 0.0239 & 0.0229 & 0.0221 & 0.0215 \\
0.5 & 0.0215 & 0.0207 & 0.0201 & 0.0197 \\
1 & 0.0205 & 0.0199 & 0.0193 & 0.0189 \\
2 & 0.0197 & 0.0191 & 0.0186 & 0.0182 \\
10 & 0.0187 & 0.0183 & 0.0178 & 0.0175 \\
\hline
\end{tabular}

TABLe 7: First nondimensional natural frequencies $₫$ of $\mathrm{ZrO}_{2} /$ SUS304 plates depending on $k_{w}^{*}(T=500 \mathrm{~K})$.

\begin{tabular}{lccccc}
\hline$N$ & \multicolumn{5}{c}{$k_{w}^{*}=100$} \\
& $k_{s}^{*}=0$ & $k_{s}^{*}=5$ & $k_{s}^{*}=10$ & $k_{s}^{*}=50$ & $k_{s}^{*}=100$ \\
\hline 0 & 0.0192 & 0.0207 & 0.0221 & 0.0311 & 0.0395 \\
0.5 & 0.0177 & 0.0190 & 0.0201 & 0.0279 & 0.0352 \\
1 & 0.0170 & 0.0182 & 0.0193 & 0.0266 & 0.0335 \\
2 & 0.0165 & 0.0176 & 0.0186 & 0.0255 & 0.0320 \\
10 & 0.0159 & 0.0169 & 0.0178 & $0.0242^{\prime}$ & 0.0303 \\
\hline
\end{tabular}

TABLE 8: First nondimensional natural frequencies $₫$ of $\mathrm{ZrO}_{2} /$ SUS304 plates depending on $k_{s}^{*}(T=500 \mathrm{~K})$.

\begin{tabular}{lccccc}
\hline$N$ & \multicolumn{5}{c}{$k_{s}^{*}=10$} \\
& $k_{w}^{*}=0$ & $k_{w}^{*}=50$ & $k_{w}^{*}=100$ & $k_{w}^{*}=200$ & $k_{w}^{*}=500$ \\
\hline 0 & 0.0207 & 0.0214 & 0.0221 & 0.0234 & 0.0270 \\
0.5 & 0.0189 & 0.0196 & 0.0201 & 0.0213 & 0.0244 \\
1 & 0.0182 & 0.0188 & 0.0193 & 0.0204 & 0.0233 \\
2 & 0.0175 & 0.0181 & 0.0186 & 0.0196 & 0.0223 \\
10 & 0.0169 & 0.0174 & 0.0178 & $0.0188^{\prime}$ & 0.0213 \\
\hline
\end{tabular}

that the results gain the exact data when the mesh is $8 \times 8$. So, the proposed theory and mathematical model are verified. For all next investigations, this $8 \times 8$ mesh will be employed.
Next, natural frequencies of FGM plates resting on elastic foundations are compared in Table 3, where Hashemi et al. [41] used the analytical approach. The FGM $\left(\mathrm{Al} / \mathrm{ZrO}_{2}\right)$ plates with dimensions $a=0.2 \mathrm{~m}$ and $b=a / 1.5$ and the plate thickness $h=a / 10$ and $h=a / 20$ are considered. Material properties $\quad E_{c}=200 \mathrm{GPa}, \quad v_{c}=0.3, \quad \rho_{c}=5700 \mathrm{~kg} / \mathrm{m}^{3}$, $E_{m}=70 \mathrm{GPa}, v_{m}=0.3$, and $\rho_{m}=2702 \mathrm{~kg} / \mathrm{m}^{3}$. The plate is fully simply supported (SSSS). Two nondimensional elastic foundation parameters are calculated as follows:

$$
\left\{\begin{array}{l}
k_{w}^{*}=\frac{K_{w} a^{4}}{A} \\
k_{s}^{*}=\frac{K_{s} a^{2}}{A}
\end{array}\right.
$$

with

$$
A=\frac{h^{3}}{\left(1-v_{m}^{2}\right)}\left[\frac{n\left(8+3 n+n^{2}\right) E_{m}+3\left(2+n+n^{2}\right) E_{c}}{12(1+n)(2+n)(3+n)}\right] .
$$

The first nondimensional natural frequency is defined as

$$
\omega^{*}=\omega h \sqrt{\frac{\rho_{c}}{E_{c}}} .
$$

It can be obtained that, from Table 3, numerical results of this work and [41] meet a good agreement. Also note that the publication [41] used the first-order shear deformation theory, and this work employs the third-order shear deformation theory; therefore, the errors shown in Table 3 can be easily understood and accepted, where the results calculated by this work are greater than or equal to results calculated by the first-order shear deformation theory [41].

Finally, nondimensional natural frequencies of FGM $\left(\mathrm{Si}_{3} \mathrm{~N}_{4} / \mathrm{SUS} 304\right)$ plate in thermal environments are compared. The geometrical dimensions are $a=b=0.2 \mathrm{~m}$, and the plate thickness $h=a / 10$. Material properties are given in Table 1 . Table 4 presents nondimensional natural frequencies of the fully clamped plate in the range of $400 \mathrm{~K}$ to $600 \mathrm{~K}$. It can be seen that this work and references [31, 42] meet a good agreement.

4.1.2. Numerical Results of Free Vibration of Piezoelectric FGM Plates Resting on Elastic Foundation in Thermal Environment. In this section, the effects of geometrical and material properties on natural frequencies of piezoelectric FGM plates are investigated. Consider a square plate with dimensions $a=0.2 \mathrm{~m}$ and the plate thickness $h=10 \mathrm{~mm}$; the 


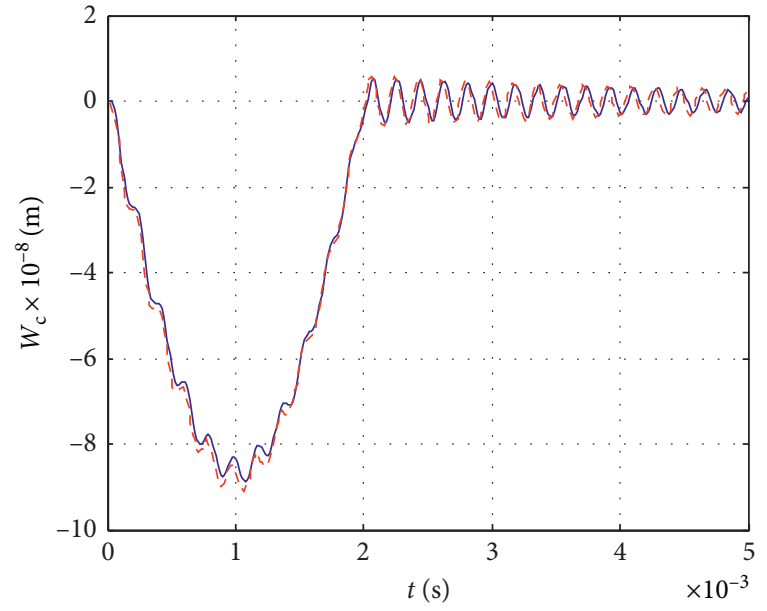

_ This work

$---[43]$

FIgURE 3: Comparison of the center point deflection changing by the time of the piezoelectric FGM plate, $n=2$.

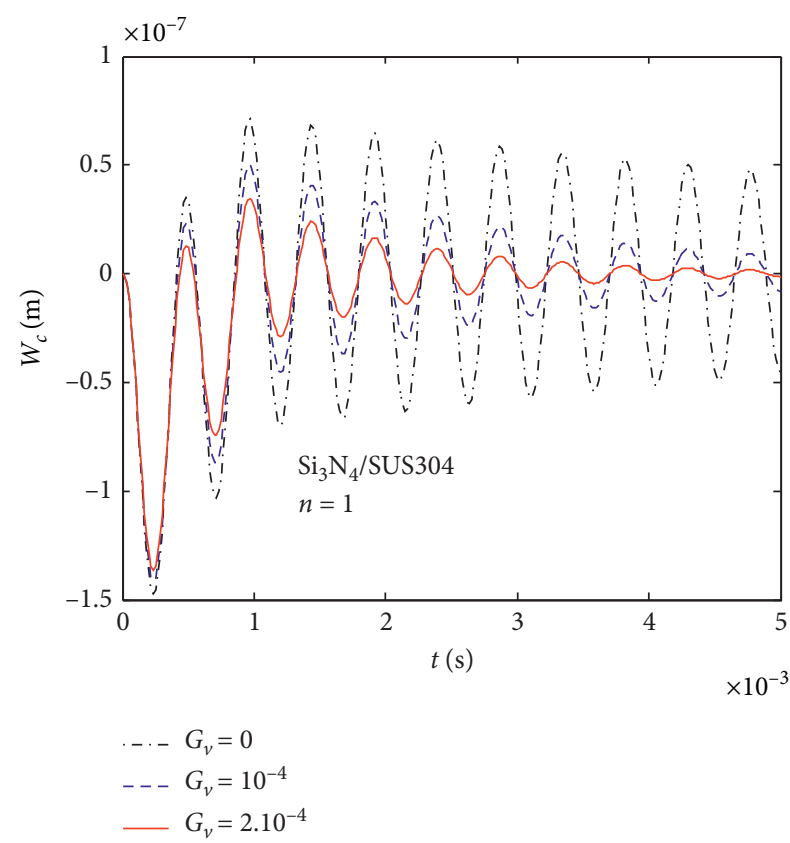

Figure 4: The dependence of $w_{c}$ on $G_{v}, n=1, T=300 \mathrm{~K}$, SSSS.

thicknesses of piezoelectric layers are $h_{a}=h_{s}=1 \mathrm{~mm}$. The FGM core layer is made of $\mathrm{Si}_{3} \mathrm{~N}_{4} / \mathrm{SUS} 304$ và $\mathrm{ZrO}_{2} / \mathrm{SUS} 304$ with material properties depending on temperature, as shown in Table 1. The plate is fully simply supported (SSSS). The nondimensional natural frequency is defined as follows:

$$
\bar{\omega}=\omega h \sqrt{\frac{\rho_{0}}{E_{0}}},
$$

where $\rho_{0}$ and $E_{0}$ are, respectively, mass density and Young modulus of $\mathrm{Si}_{3} \mathrm{~N}_{4} / \mathrm{SUS} 304$ at $300 \mathrm{~K}$.

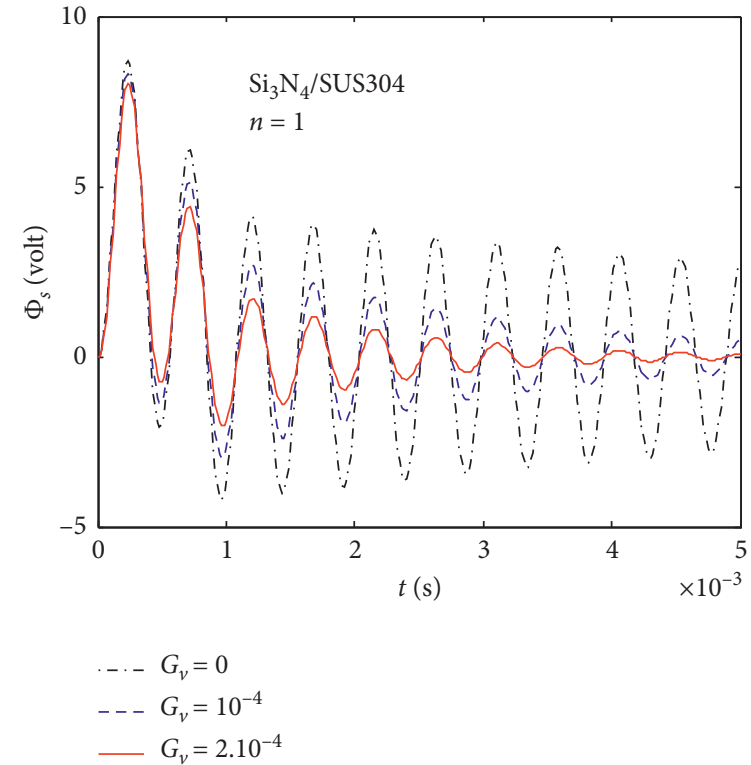

Figure 5: The dependence of $\phi_{s}$ on $G_{v}, n=1, T=300 \mathrm{~K}$, SSSS.

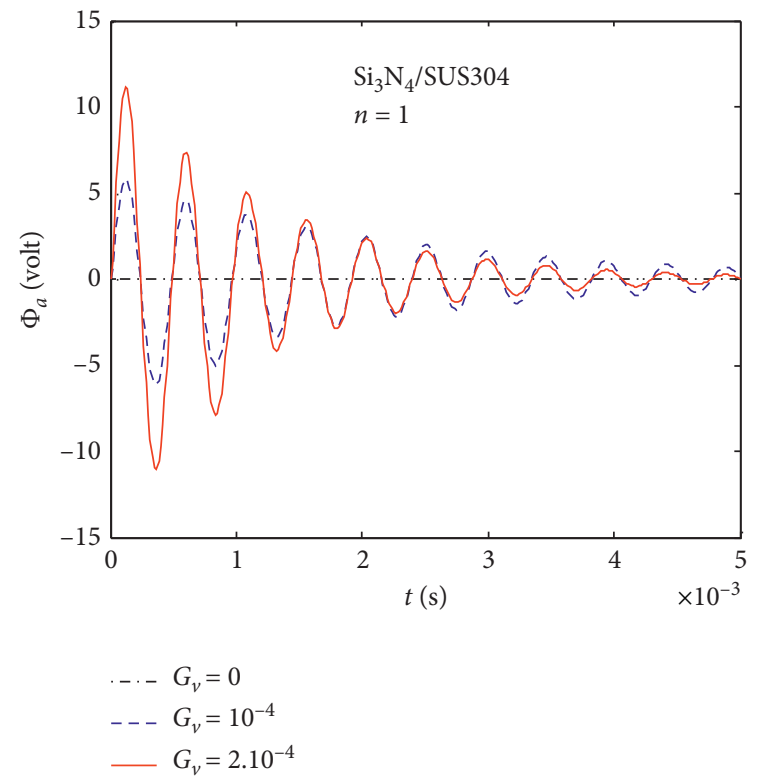

Figure 6: The dependence of $\phi_{a}$ on $G_{v}, n=1, T=300 \mathrm{~K}$, SSSS.

By changing the temperature in a range of $300 \mathrm{~K}$ to $600 \mathrm{~K}$, nondimensional natural frequencies $\bar{\omega}$ of the plate are presented in Tables 5 and 6 . As changing the stiffness coefficient of the elastic foundation, nondimensional natural frequencies $\bar{\omega}$ are shown in Tables 7 and 8 . Based on numerical results, some comments are given as follows.

When increasing the temperature, the nondimensional natural frequency of the plate decreases for all values of the volume fraction index. The reason is that Young's modulus of the core layer decreases on increasing the temperature while the mass density is remained; therefore, the first nondimensional natural frequency of the plate will decrease. 


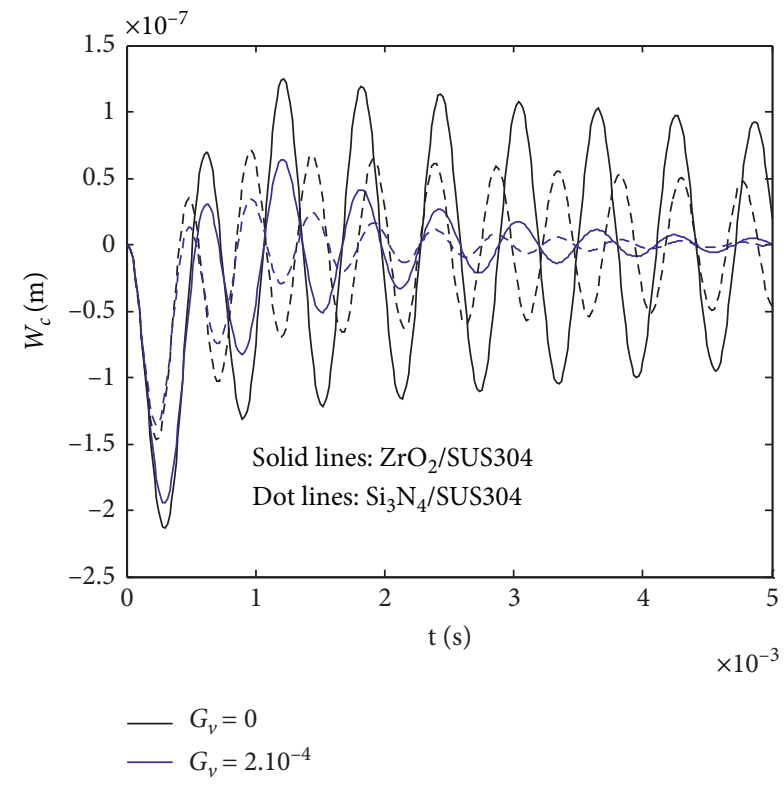

FIGURE 7: The dependence of $w_{c}$ on $G_{v}$ with two FG materials, $n=1$, $T=300 \mathrm{~K}$, SSSS.

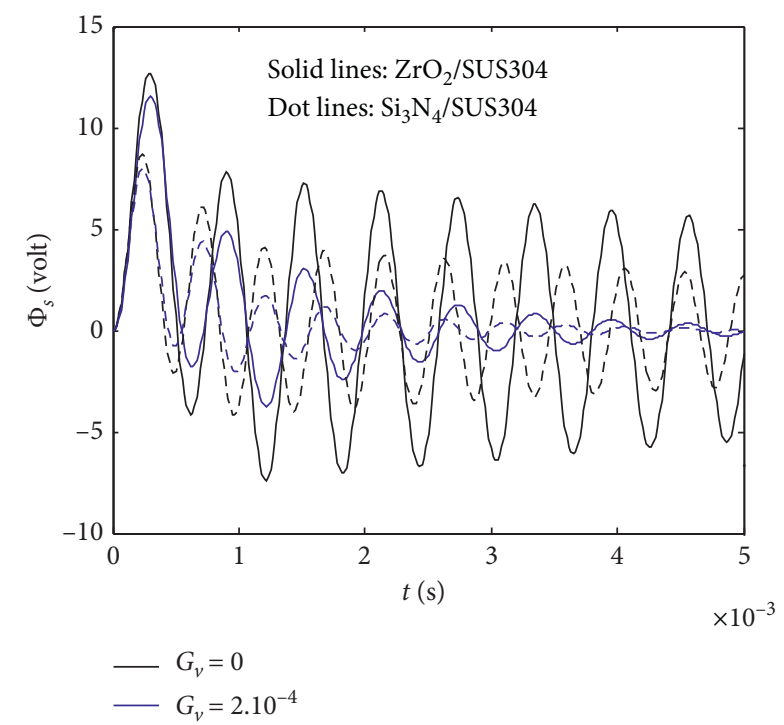

Figure 8: The dependence of $\phi_{s}$ on $G_{v}$ with two FG materials, $n=1$, $T=300 \mathrm{~K}$, SSSS.

At the same time, when increasing the volume fraction index $n$, the proportion of the metal increases, which leads to the decrease in the total mass of the structure; therefore, the natural frequencies of this FGM plate decrease.

On increasing the stiffness coefficient of the elastic foundation, the stiffness matrix of the whole plate will be added by the addition stiffness component of the elastic foundation, and hence, the first nondimensional natural frequency of the plate will increase.

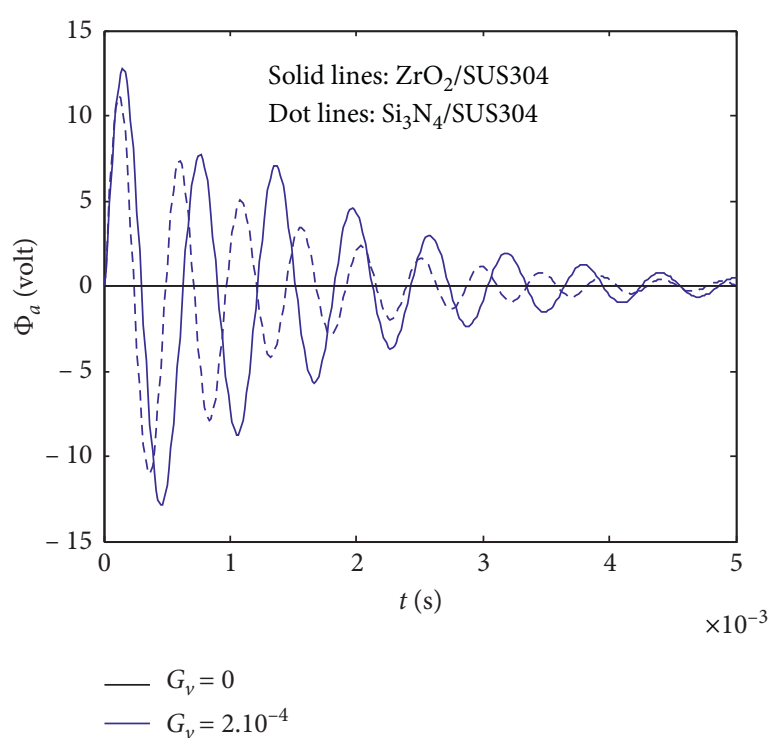

FIgURE 9: The dependence of $\phi_{a}$ on $G_{v}$ with two FG materials, $n=1$, $T=300 \mathrm{~K}$, SSSS.

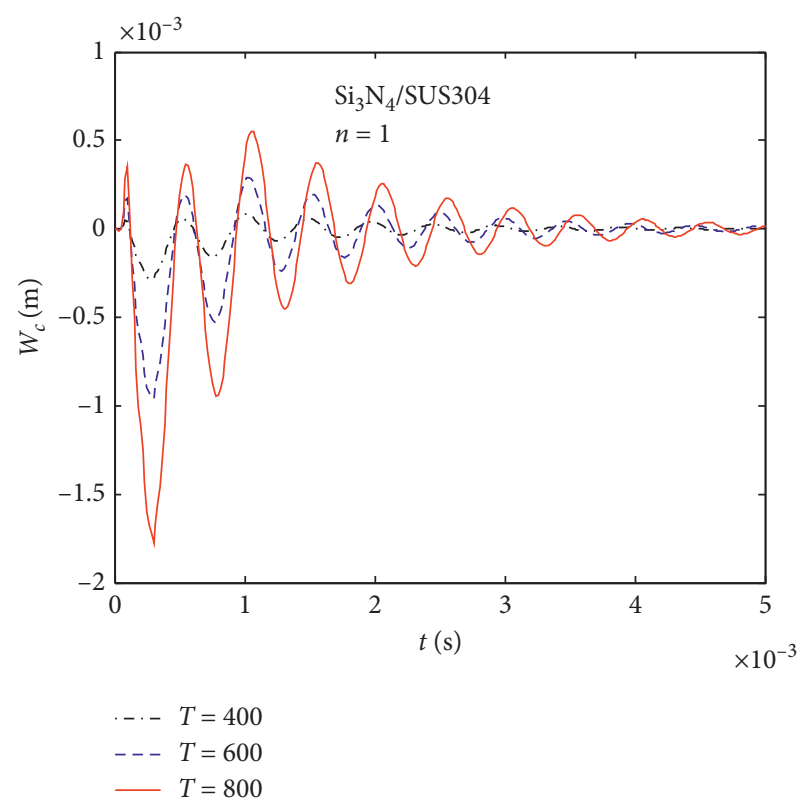

FIGURE 10: The dependence of $w_{c}$ on the temperature, $n=1$, SSSS, $k_{1}^{*}=100, k_{2}^{*}=10, G_{v}=2.10^{-4}$.

\subsection{Numerical Results of Dynamic Response for Sandwich FGM Plates in High Temperature}

4.2.1. Accuracy Study for Dynamic Response of Piezoelectric FGM Plates. Consider a square plate with $a=b=0.2 \mathrm{~m}$, the plate thickness $20 \mathrm{~mm}$; the piezoelectric layer has a thickness of $2 \mathrm{~mm}$. The plate is fully clamped. The core layer is made of Ti-6Al-4 V/Aluminum oxide, and the piezoelectric face sheet is shown in Section 4.1.1. The load changing by time is described as 


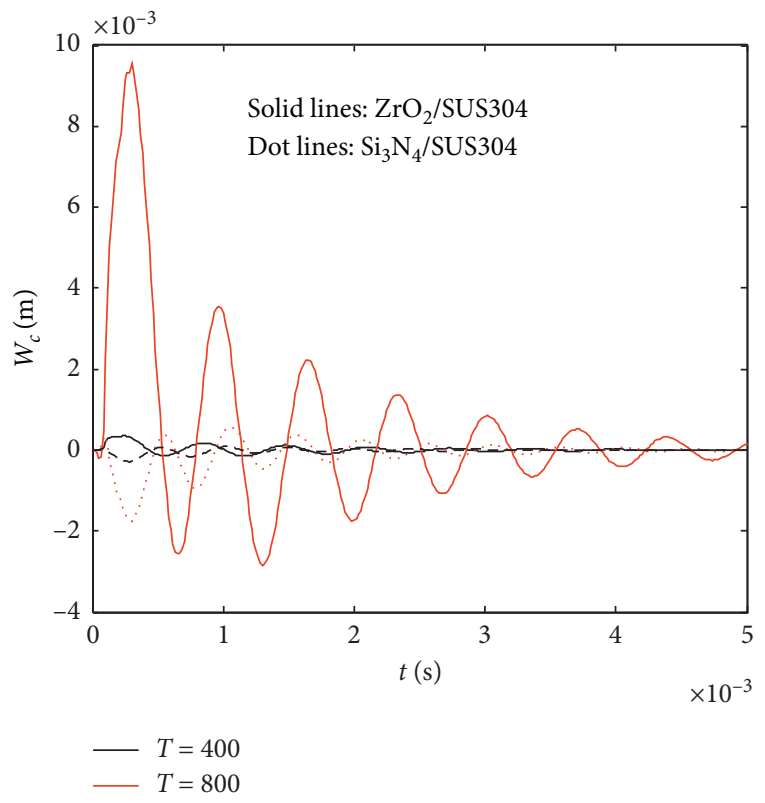

FIgURE 11: The dependence of $w_{c}$ on the temperature with two FG materials, $n=1$, SSSS, $k_{1}^{*}=100, k_{2}^{*}=10, G_{v}=2.10^{-4}$.

TABle 9: Maximum deflection $w_{c}$ ( $\left.\mathrm{mm}\right)$ depending on $n$ and temperature, $\mathrm{Si}_{3} \mathrm{~N}_{4} / \mathrm{SUS} 304, G_{v}=2.10^{-4}$.

\begin{tabular}{lccccc}
\hline & $T=300 \mathrm{~K}$ & $400 \mathrm{~K}$ & $500 \mathrm{~K}$ & $600 \mathrm{~K}$ & $800 \mathrm{~K}$ \\
\hline$n=0$ & 0.000124 & 0.0933 & 0.2000 & 0.3198 & 0.5989 \\
$n=0.5$ & 0.000133 & 0.2330 & 0.4937 & 0.7812 & 1.4508 \\
$n=1$ & 0.000136 & 0.2858 & 0.6045 & 0.9578 & 1.7772 \\
$n=2$ & 0.000138 & 0.2949 & 0.6229 & 0.9804 & 1.8144 \\
$n=5$ & 0.000140 & 0.2324 & 0.4893 & 0.7737 & 1.4225 \\
$n=10$ & 0.000141 & 0.1735 & 0.3700 & 0.5904 & 1.1011 \\
\hline
\end{tabular}

$$
P=P_{0} \sin \left(\frac{\pi x}{a}\right) \sin \left(\frac{\pi y}{b}\right) F(t),
$$

with $P_{0}=10^{3} \mathrm{~N} / \mathrm{m}^{2}$, and the function $F(t)$ has the following form:

$$
F(t)= \begin{cases}\sin \left(\pi \frac{t}{t_{1}}\right), & 0 \leq t \leq t_{1} \\ 0, & t>t_{1},\end{cases}
$$

where $t_{1}=1 \mathrm{~ms}$.

The dynamic responses of the center point of the piezoelectric FGM plate changing by the time are presented in Figure 3 and compared with those of the cell-based smoothed discrete shear gap method [43]. It can be observed that they meet a good agreement; therefore, the proposed theory and mathematical model are verified.

4.2.2. Numerical Results for Dynamic Response of Piezoelectric FGM Plates in High Temperature. This section investigates the effects of some geometrical and material properties on the dynamic response of piezoelectric sandwich
TABLE 10: Maximum deflection $w_{c}(\mathrm{~mm})$ depending on $n$ and temperature, $\mathrm{ZrO}_{2} / \mathrm{SUS} 304, G_{v}=2 \cdot 10^{-4}$.

\begin{tabular}{lccccc}
\hline & $T=300 \mathrm{~K}$ & $400 \mathrm{~K}$ & $500 \mathrm{~K}$ & $600 \mathrm{~K}$ & $800 \mathrm{~K}$ \\
\hline$n=0$ & 0.0002063 & 0.3169 & 0.8967 & 1.8400 & 5.2122 \\
$n=0.5$ & 0.0001976 & 0.3323 & 1.1231 & 2.5868 & 8.1864 \\
$n=1$ & 0.000194 & 0.3638 & 1.2806 & 2.9506 & 9.5619 \\
$n=2$ & 0.000191 & 0.3534 & 1.2216 & 2.9051 & 9.3896 \\
$n=5$ & 0.000188 & 0.2681 & 0.8920 & 2.0752 & 6.6588 \\
$n=10$ & 0.000186 & 0.2070 & 0.6197 & 1.3717 & 4.3010 \\
\hline
\end{tabular}

FGM plates resting on two-parameter elastic foundations, where the whole structure is placed in the thermal environment. Consider a fully simply supported square plate with $a=b=0.2 \mathrm{~m}$, the plate thickness $h=10 \mathrm{~mm}$. The core layer is made of functionally graded materials $\mathrm{Si}_{3} \mathrm{~N}_{4} / \mathrm{SUS} 304$ and $\mathrm{ZrO}_{2} / \mathrm{SUS} 304$ where the material properties are shown in Table 1. Two face sheets are the piezoelectric G-1195N material with the thicknesses $h_{a}=h_{s}=1 \mathrm{~mm}$, and mechanical properties are $E_{p i}=63 \mathrm{GPa}$, Poison's ratio $v_{p i}=0.3$, $d_{31}=d_{32}=254.10^{-12} \mathrm{~m} / \mathrm{V}$, and $k_{33}=15 \mathrm{nF} / \mathrm{m}$. The plate is subjected to the triangular load with the form as shown in equation (44) with the function $F(t)$ in the following form:

$$
F(t)= \begin{cases}1-\frac{t}{t_{1}}, & 0 \leq t \leq t_{1}, \\ 0, & t>t_{1} .\end{cases}
$$

Nondimensional elastic foundation parameters are defined as

$$
\left\{\begin{array}{l}
k_{1}^{*}=\frac{K_{w} a^{4}}{D}, \\
k_{2}^{*}=\frac{K_{s} a^{2}}{D},
\end{array}\right.
$$

with

$$
D=\frac{E_{c} h^{3}}{12\left(1-v_{c}^{2}\right)}
$$

The output results are the deflection $w$ and voltages $\phi_{s}, \phi_{a}$ at the center point of the plate. The Newmark- $\beta$ is used to solve the dynamic equation with parameters $\bar{\alpha}$ and $\bar{\beta}$ having the values of 0.5 and 0.25 , respectively.

(1) Effect of Feedback Coefficient. $G_{v}$ To see more clearly the effect of the feedback coefficient $G_{v}$ on the ability to reduce the oscillation of the plate, this subsection considers a piezoelectric FGM plate with the volume fraction index $n=1$. The parameters of the two-parameter elastic foundation $k_{1}^{*}=100$ and $k_{2}^{*}=10$. The results of the deflection $w$ and the voltages $\phi_{s}, \phi_{a}$ at the center point of the plate are presented in Figures 4-9. Some comments are as follows.

When increasing the feedback coefficient $G_{v}$, the deflection $w$ of the plate is reduced rapidly; in other words, the ability to reduce the oscillation of the plate is better. 


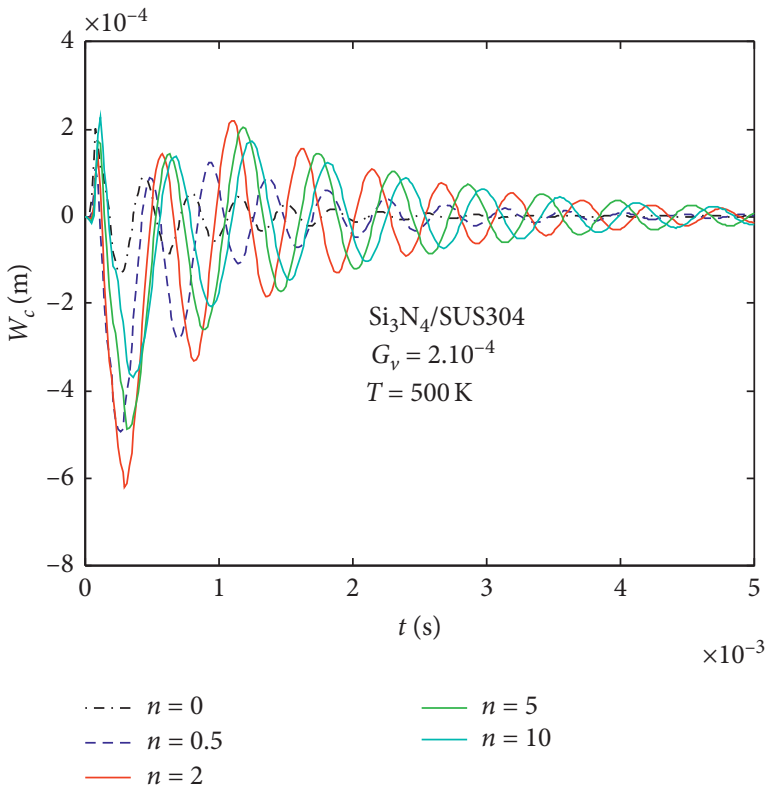

FIgURE 12: The dependence of $w_{c}$ on $n, \mathrm{Si}_{3} \mathrm{~N}_{4} / \mathrm{SUS} 304$, SSSS, $k_{1}^{*}=100, k_{2}^{*}=10, G_{v}=2.10^{-4}$.

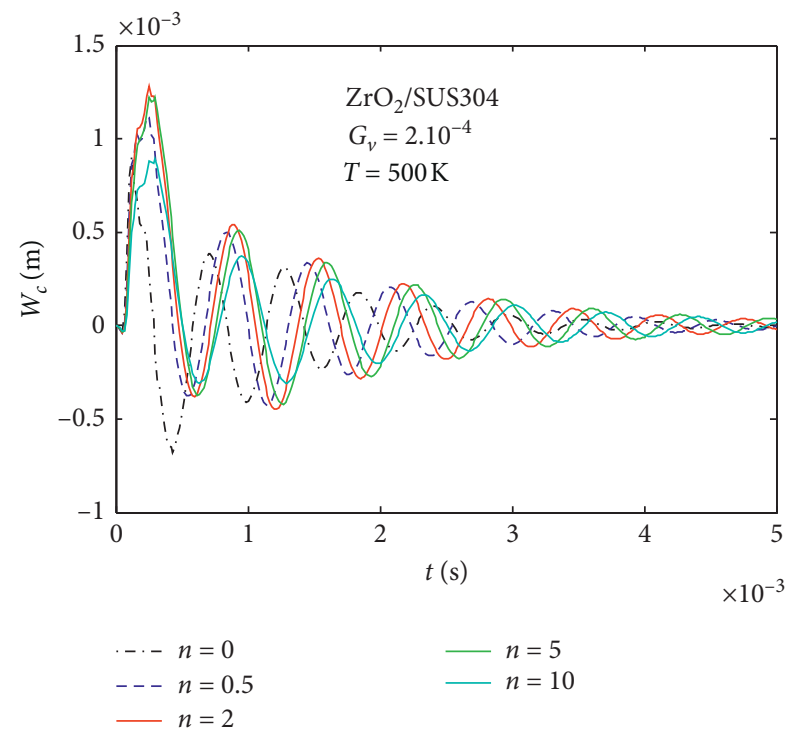

FIGURE 13: The dependence of $w_{c}$ on $n, \mathrm{ZrO}_{2} / \mathrm{SUS} 304$, SSSS, $k_{1}^{*}=100, k_{2}^{*}=10, G_{v}=2.10^{-4}$.

Increasing the feedback coefficient $G_{v}$, the voltages $\phi_{s}$ and $\phi_{a}$ are also reduced quickly; however, when $G_{v}$ gets higher values, the voltage $\phi_{s}$ decreases and $\phi_{a}$ increases. That is because $\phi_{s}$ is proportional to the nodal displacement according to equation (30); however, the displacement of the plate should also decrease, and $\phi_{a}$ is proportional to $G_{v}$, so in the first stage, when the external force is active, $\phi_{a}$ increases when $G_{v}$ increases, and $\phi_{a}$ also decreases rapidly when out of force. Besides, from Figures 4 and 5, it can be seen that when increasing the value of $G_{v}$, the maximum deflection $w_{c}$ and $\phi_{s}$ in the active external phase do not decrease much, but when the external force is not acting on the structure (free

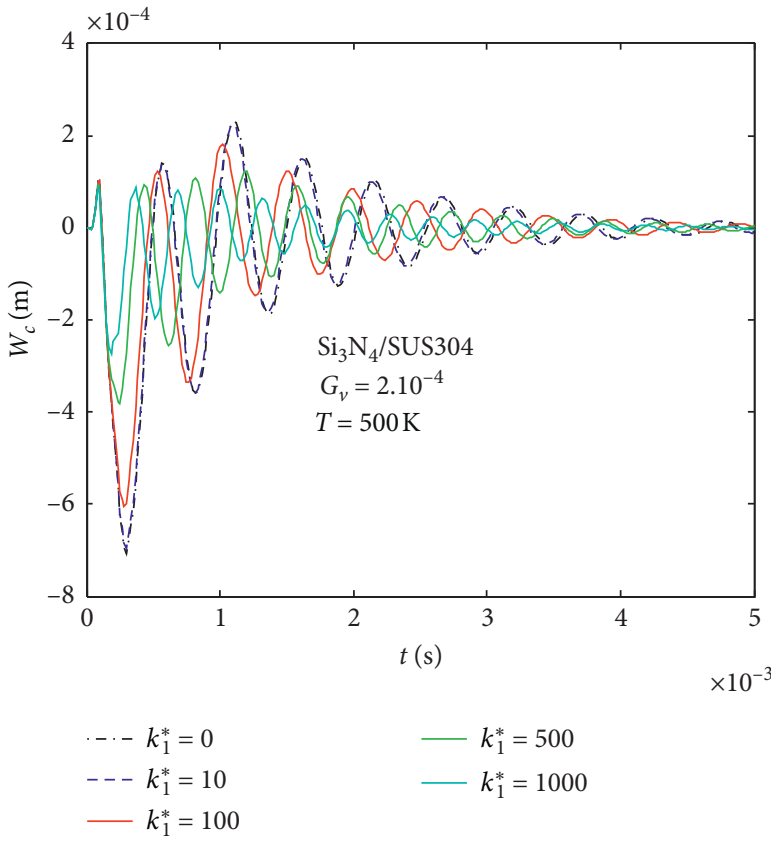

FIgURE 14: The dependence of $w_{c}$ on $k_{1}^{*}, \mathrm{Si}_{3} \mathrm{~N}_{4} / \mathrm{SUS} 304, \mathrm{SSSS}$, $k_{2}^{*}=10, G_{v}=2.10^{-4}$.

oscillation phase), the maximum value of deflection $w_{c}$ and $\phi_{s}$ decreases significantly and the dimming time of these components also decreases rapidly as $G_{v}$ increases. It is explained that when the value of $G_{v}$ increases, the drag coefficient of the structure related to $G_{v}$ also increases, and this leads to the loss of the total energy of the structure; therefore, the oscillation of the plate is rapidly extinguished. This also indicates that, in practice, to control plate oscillation, if piezoelectric layers with appropriate $G_{v}$ coefficients are used, it will increase the working capacity of the structure.

(2) Effect of Temperature. When increasing the temperature in a range of $400 \mathrm{~K}$ to $800 \mathrm{~K}$, the deflection $w_{c}$ of the center point of the plate $\left(\mathrm{Si}_{3} \mathrm{~N}_{4} / \mathrm{SUS} 304\right.$ and $\left.\mathrm{ZrO}_{2} / \mathrm{SUS} 304\right)$ changing by the time is presented in Figures 10 and 11. It can see clearly that the temperature has a strong effect on the dynamic response of the structure. At the same time, the temperature is the main reason for changing the mechanical properties of the material, and the frequencies of the plate vary; as a result, the time of maximum occurrence of deflection $w_{c}$ is not the same. In addition, as the temperature increases, the frequency of the plate changes; hence, the shape of the response line of the plate corresponding to different temperature cases is also changed, which causes the response curves to be inconsistent with each other. Thus, in practice, when the plate is working in a high-temperature environment, piezoelectric layers can be used to control the vibration of this structure according to the requirements.

(3) Effect of Volume Fraction Index. Next, the effect of the volume fraction index $n$ is examined in this section. The maximum deflection $w_{c}$ of the center point of the plate for 


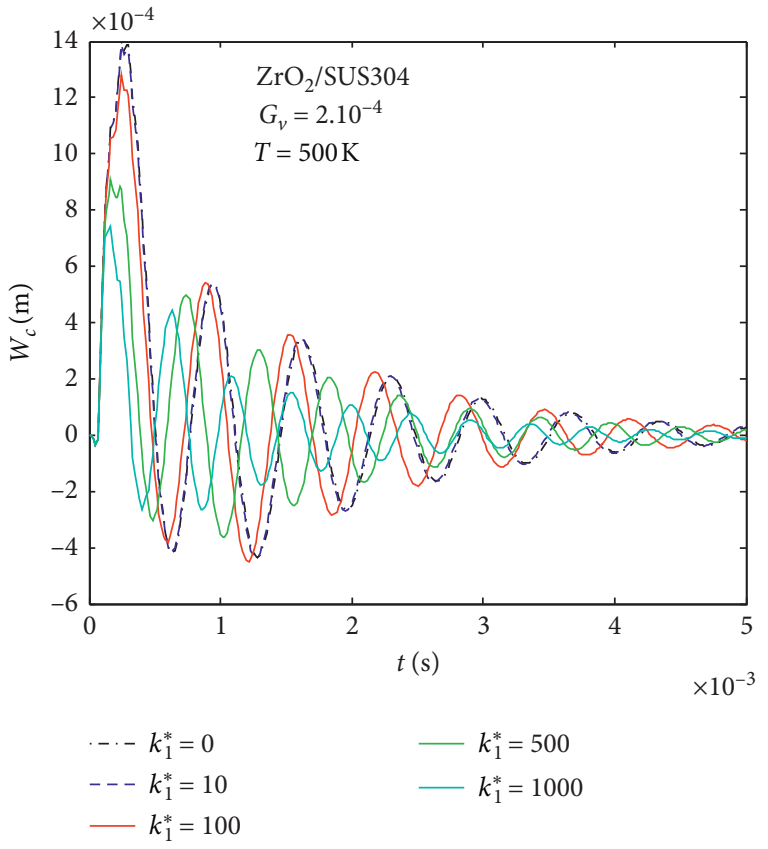

FIgURE 15: The dependence of $w_{c}$ on $k_{1}^{*}, \mathrm{ZrO}_{2} / \mathrm{SUS} 304$, SSSS, $k_{2}^{*}=10, G_{v}=2 \cdot 10^{-4}$.

the case of $n$ changing in a range of 0 to 10 is presented in Tables 9 and 10. The deflection changing by the time is shown in Figures 12 and 13. It can be obtained that, for the case of $T=300 \mathrm{~K}$, when increasing the volume fraction index $n$, the maximum deflection increases. However, for the case of $T>300 \mathrm{~K}$, the value, as well as the shape of the deflection $w_{c}$, will change by the time, and as a result, the plate obtains the maximum deflection at different moments. Besides, the maximum deflection increases when $n$ is small, and then, it decreases as $n$ gets higher values. This is because as the volume fraction index $n$ increases, the metal ratio increases, and the stiffness of the plate decreases.

(4) Effect of Elastic Foundation. Now, the effect of the elastic foundation is investigated. This case $k_{2}^{*}=10$, let $k_{1}^{*}$ increase gradually from 0 to 1000 , and the dependence of the center point deflection on $k_{1}^{*}$ is shown in Figures 14 and 15. It can be seen that when increasing the value of $k_{1}^{*}$, the maximum deflection of the plate decreases. It is explained that when increasing the value of the elastic foundation coefficient, the total energy of the plate is added by the energy relative to the elastic foundation coefficient, which increases the stiffness of the structure, so the maximum deflection of the plate is increased. Besides, the shape of the deflection response is also changed by the stiffness of the elastic foundation, this is due to the change in the stiffness of the plate, and the frequency of the plate is also changed.

\section{Conclusions}

This paper presents new free and forced vibrations of piezoelectric FGM plates resting on two-parameter elastic foundations placed in thermal environments. Finite element formulations are established by using the third-order shear deformation theory of Reddy and the finite element method. The reliability of the proposed theory and mathematical model is verified by comparing the results of this work with those of other methods. A series of parameter studies are conducted to examine the effects of geometrical and mechanical properties of the plates on free and forced vibrations of piezoelectric FGM plates. Some remarkable points are concluded as follows:

(1) When increasing the temperature, the first natural frequency of the plate is reduced, and this is because the elastic modulus of both metal and ceramic is reduced; therefore, the deflection of the plate under forced loads increases when the temperature increases, and the time response curves are also not uniform.

(2) When increasing the feedback coefficient $G_{v}$, during the period of forced oscillation, the maximum deflection of the plate does not decrease much, but when the external force is not active, the maximum deflection of the plate decreases rapidly. This is the best advantage of the structure when adding piezoelectric components.

(3) When increasing the stiffness of the elastic foundation and the value of the volume fraction index $n$, the maximum deflection of the plate is reduced.

The results in this study are the reference to apply in the vibration control of piezoelectric FGM plates resting on elastic foundations in high-temperature environments. Depending on the practical requirements, it can be set out, the appropriate coefficient $G_{v}$ is selected, and we will obtain the desired deflection value, which increases the working capacity for the structure. At the same time, this is one of the solutions that contribute to the optimization of structures according to engineering requirements.

\section{Data Availability}

The data used to support the findings of this study are included within the article.

\section{Conflicts of Interest}

The authors declare that there are no conflicts of interest regarding the publication of this paper.

\section{Acknowledgments}

This work was supported by the University of Transport and Communications Foundation for Science and Technology Development (Grant no. 786/TB-DHGTVT).

\section{References}

[1] B. N. Lieu, V. N. Nam, H. T. Chien, A. M. J. Ferreira, and N. X. Hung, "An isogeometric Bézier finite element analysis for piezoelectric FG porous plates reinforced by graphene platelets," Composite Structures, vol. 214, pp. 227-245, 2019. 
[2] M. R. Barati, H. Shahverdi, and A. M. Zenkour, "Electromechanical vibration of smart piezoelectric FG plates with porosities according to a refined four-variable theory," $M e$ chanics of Advanced Materials and Structures, vol. 24, no. 12, pp. 987-998, 2017.

[3] V. N. Nam, L. Jaehong, and N. X. Hung, "Active vibration control of GPLs-reinforced FG metal foam plates with piezoelectric sensor and actuator layers," Composites Part B: Engineering, vol. 172, pp. 769-784, 2019.

[4] M. M. Keleshteri, H. Asadi, and M. M. Aghdam, "Geometrical nonlinear free vibration responses of FG-CNT reinforced composite annular sector plates integrated with piezoelectric layers," Composite Structures, vol. 171, pp. 100-112, 2017.

[5] P. Malekzadeh, A. R. Setoodeh, and M. Shojaee, "Vibration of FG-GPLs eccentric annular plates embedded in piezoelectric layers using a transformed differential quadrature method," Computer Methods in Applied Mechanics and Engineering, vol. 340, pp. 451-479, 2018.

[6] F. Ebrahimi and A. Rastgoo, "Free vibration analysis of smart annular FGM plates integrated with piezoelectric layers," Smart Materials and Structures, vol. 17, no. 1, p. 13, Article ID 015044, 2008

[7] M. M. Keleshteri, H. Asadi, and Q. Wang, "On the snapthrough instability of post-buckled FG-CNTRC rectangular plates with integrated piezoelectric layers," Computer Methods in Applied Mechanics and Engineering, vol. 331, pp. 53-71, 2018.

[8] M. M. Keleshteri, H. Asadi, and Q. Wang, "Postbuckling analysis of smart FG-CNTRC annular sector plates with surface-bonded piezoelectric layers using generalized differential quadrature method," Computer Methods in Applied Mechanics and Engineering, vol. 325, pp. 689-710, 2017.

[9] A. A. Hassen, T. Abdelouahed, M. Ismail, and E. A. A. Bedia, "Free vibration analysis of functionally graded plates resting on Winkler-Pasternak elastic foundations using a new shear deformation theory," International Journal of Mechanics and Materials in Design, vol. 6, pp. 113-121, 2010.

[10] D. Q. Chan, N. Van Thanh, N. D. Khoa, and N. D. Duc, "Nonlinear dynamic analysis of piezoelectric functionally graded porous truncated conical panel in thermal environments," Thin-Walled Structures, vol. 154, p. 106837, 2020.

[11] A. M. Zenkour and A. F. Radwan, "Bending response of FG plates resting on elastic foundations in hygrothermal environment with porosities," Composite Structures, vol. 213, pp. 133-143, 2019.

[12] H.-S. Shen and Y. Xiang, "Postbuckling of axially compressed nanotube-reinforced composite cylindrical panels resting on elastic foundations in thermal environments," Composites Part B: Engineering, vol. 67, pp. 50-61, 2014.

[13] D. G. Ninh, "Nonlinear thermal torsional post-buckling of carbon nanotube-reinforced composite cylindrical shell with piezoelectric actuator layers surrounded by elastic medium," Thin-Walled Structures, vol. 123, pp. 528-538, 2018.

[14] M. Nebab, A. A. Hassen, R. Bennai, A. Tounsi, and E. A. A. Bedia, "Vibration response and wave propagation in FG plates resting on elastic foundations using HSDT," Structural Engineering \& Mechanics, vol. 69, no. 5, pp. 511525, 2019.

[15] N. D. Duc and T. Q. Quan, "Nonlinear stability analysis of double-curved shallow FGM panels on elastic foundations in thermal environments," Mechanics of Composite Materials, vol. 48, no. 4, pp. 435-448, 2012.

[16] S.-E. Kim, N. D. Duc, V. H. Nam, and N. Van Sy, "Nonlinear vibration and dynamic buckling of eccentrically oblique stiffened FGM plates resting on elastic foundations in thermal environment," Thin-Walled Structures, vol. 142, pp. 287-296, 2019.

[17] T. V. Do, T. Q. Bui, T. T. Yu, D. T. Pham, and C. T. Nguyen, "Role of material combination and new results of mechanical behavior for FG sandwich plates in thermal environment," Journal of Computational Science, vol. 21, pp. 164-181, 2017.

[18] B. T. T. Mai, N. H. Cuong, N. D. Quang, and D. H. Tai, "Experimental study on flexural and shear behaviour of sandwich panels using glass textile reinforced concrete and autoclaved aerated concrete," Transport and Communications Science Journal, vol. 71, no. 1, pp. 18-26, 2020.

[19] R. Abdelkrim, A. Chikh, T. Abdelouahed et al., "Physical stability response of a SLGS resting on viscoelastic medium using nonlocal integral first-order theory," Steel and Composite Structures, vol. 37, no. 6, pp. 695-709, 2020.

[20] B. Noureddine, M. Zidour, A. B. Abdelmoumen et al., "Deflections, stresses and free vibration studies of FG-CNT reinforced sandwich plates resting on Pasternak elastic foundation," Computers and Concrete, vol. 26, no. 3, pp. 213-226, 2020.

[21] M. Rabhi, H. B. Kouider, K. Abdelhakim et al., "A new innovative 3-unknowns HSDT for buckling and free vibration of exponentially graded sandwich plates resting on elastic foundations under various boundary conditions," Geomechanics and Engineering, vol. 22, no. 2, pp. 119-132, 2020.

[22] B. Fouad, A. B. Abdelmoumen, T. Abdeldjebbar et al., "Stability and dynamic analyses of SW-CNT reinforced concrete beam resting on elastic-foundation," Computers and Concrete, vol. 25, no. 6, pp. 485-495, 2020.

[23] K. Mokhtar, A. B. Abdelmoumen, K. Abdelhakim, B. Aicha, T. Abdelouahed, and S. R. Mahmoud, "Analytical modeling of bending and vibration of thick advanced composite plates using a four-variable quasi 3D HSDT," Engineering with Computers, vol. 36, pp. 807-821, 2020.

[24] K. Miloud, K. Abdelhakim, A. B. Abdelmoumen et al., "A study on the structural behaviour of functionally graded porous plates on elastic foundation using a new quasi-3D model: bending and Free vibration analysis," Computers and Concrete, vol. 25, no. 1, pp. 37-57, 2020.

[25] Y. A. Farouk, M. Mustapha, A. B. Abdelmoumen et al., "Influences of porosity on dynamic response of FG plates resting on Winkler/Pasternak/Kerr foundation using quasi 3D HSDT," Computers and Concrete, vol. 24, no. 4, pp. 347-367, 2019.

[26] N. T. Hong, "Nonlinear static bending and free vibration analysis of bidirectional functionally graded material plates," International Journal of Aerospace Engineering, vol. 2020, Article ID 8831366, 16 pages, 2020.

[27] R. Salah, A. B. Abdelmoumen, B. Abdelhakim et al., "Effects of hygro-thermo-mechanical conditions on the buckling of FG sandwich plates resting on elastic foundations," Computers and Concrete, vol. 25, no. 4, pp. 311-325, 2020.

[28] T. Abdelouahed, S. U. Al-Dulaijan, M. A. Al-Osta et al., "A four variable trigonometric integral plate theory for hygrothermo-mechanical bending analysis of AFG ceramic-metal plates resting on a two-parameter elastic foundation," Steel and Composite Structures, vol. 34, no. 4, pp. 511-524, 2020.

[29] M. Hakima, A. B. Abdelmoumen, H. Houari et al., "Investigation on hygro-thermal vibration of P-FG and symmetric S-FG nanobeam using integral Timoshenko beam theory," Advances in Nano Research, vol. 8, no. 4, pp. 293-305, 2020.

[30] B. Abderrafik, B. Belhadj, B. Mohamed et al., "A simple nthorder shear deformation theory for thermomechanical 
bending analysis of different configurations of FG sandwich plates," Smart Structures and Systems, vol. 25, no. 2, pp. 197-218, 2020.

[31] T. Q. Bui, T. V. Do, L. H. T. Ton et al., "On the high temperature mechanical behaviors analysis of heated functionally graded plates using FEM and a new third-order shear deformation plate theory," Composites Part B: Engineering, vol. 92, pp. 218-241, 2016.

[32] J. N. Reddy, "Analysis of functionally graded plates," International Journal for Numerical Methods in Engineering, vol. 47, no. 1-3, pp. 663-684, 2000.

[33] H.-N. Nguyen, T. C. Tan, D. T. Luat, V.-D. Phan, D. V. Thom, and P. V. Minh, "Research on the buckling behavior of functionally graded plates with stiffeners based on the thirdorder shear deformation theory," Materials, vol. 12, no. 8, p. 1262, 2019.

[34] V. N. Hoai, D. H. Doan, N. M. Khoa, T. Van Do, and H. T. Tran, "Phase-field buckling analysis of cracked stiffened functionally graded plates," Composite Structures, vol. 217, pp. 50-59, 2019.

[35] P. P. Minh, "Analysis free vibration of the functionally grade material cracked plates with varying thickness using the Phase-field theory," Transport and Communications Science Journal, vol. 70, no. 2, pp. 122-131, 2019.

[36] M. Y. Yasin, N. Ahmad, and M. N. Alam, "Finite element analysis of actively controlled smart plate with patched actuators and sensors," Latin American Journal of Solids and Structures, vol. 7, no. 3, pp. 227-247, 2010.

[37] S. Valliappan and K. Qi, "Finite element analysis of a smart damper for seismic structural control," Computers and Structures, vol. 81, pp. 1009-1017, 3003.

[38] H. S. Tzou and C. I. Tseng, "Distributed piezoelectric sensor/ actuator design for dynamic measurement/control of distributed parameter systems: a piezoelectric finite element approach," Journal of Sound and Vibration, vol. 138, no. 1, pp. 17-34, 1990.

[39] X. Q. He, T. Y. Ng, S. Sivashanker, and K. M. Liew, “Active control of FGM plates with integrated piezoelectric sensors and actuators," International Journal of Solids and Structures, vol. 38, no. 9, pp. 1641-1655, 2001.

[40] M. A. A. Farsangi and A. R. Saidi, "Levy type solution for free vibration analysis of functionally graded rectangular plates with piezoelectric layers," Smart Materials and Structures, vol. 21, p. 15, Article ID 094017, 2012.

[41] S. H. Hashemi, H. R. D. Taher, H. Akhavan, and M. Omidi, "Free vibration of functionally graded rectangular plates using first-order shear deformation plate theory," Applied Mathematical Modelling, vol. 34, no. 5, pp. 1276-1291, 2010.

[42] N. Wattanasakulpong, G. B. Prusty, and D. W. Kelly, "Free and forced vibration analysis using improved third-order shear deformation theory for functionally graded plates under high temperature loading," Journal of Sandwich Structures \& Materials, vol. 15, no. 5, pp. 583-606, 2013.

[43] K. N. Quang, H. D. Trung, VH Huu, H. L. Van, and T. N. Thoi, "Analysis and control of FGM plates integrated with piezoelectric sensors and actuators using cell-based smoothed discrete shear gap method (CS-DSG3)," Composite Structures, vol. 165, pp. 115-129, 2017. 